\title{
Detecting Eccentric Supermassive Black Hole Binaries With Pulsar Timing Arrays: Resolvable Source Strategies
}

\author{
S. R. Taylor \\ E. A. Huerta \\ J. R. Gair \\ S. T. McWilliams
}

Follow this and additional works at: https://researchrepository.wvu.edu/faculty_publications

\section{Digital Commons Citation}

Taylor, S. R.; Huerta, E. A.; Gair, J. R.; and McWilliams, S. T., "Detecting Eccentric Supermassive Black Hole Binaries With Pulsar Timing Arrays: Resolvable Source Strategies" (2016). Faculty \& Staff Scholarship. 9. https://researchrepository.wvu.edu/faculty_publications/9

This Article is brought to you for free and open access by The Research Repository @ WVU. It has been accepted for inclusion in Faculty \& Staff Scholarship by an authorized administrator of The Research Repository @ WVU. For more information, please contact beau.smith@mail.wvu.edu. 


\title{
DETECTING ECCENTRIC SUPERMASSIVE BLACK HOLE BINARIES WITH PULSAR TIMING ARRAYS: RESOLVABLE SOURCE STRATEGIES
}

\author{
S. R. TAYlor ${ }^{1}$, E. A. Huerta ${ }^{2,3}$, J. R. Gair ${ }^{4,5}$, and S. T. McWilliams ${ }^{2}$ \\ ${ }^{1}$ Jet Propulsion Laboratory, California Institute of Technology, 4800 Oak Grove Drive, Pasadena, CA 91106, USA; Stephen.R.Taylor@jpl.nasa.gov \\ ${ }^{2}$ Department of Physics and Astronomy, West Virginia University, White Hall, Morgantown, WV 26506, USA \\ NCSA, University of Illinois at Urbana-Champaign, IL 61801, USA \\ ${ }_{5}^{4}$ Institute of Astronomy, University of Cambridge, Madingley Rd., Cambridge, CB3 0HA, UK \\ ${ }^{5}$ School of Mathematics, University of Edinburgh, King's Buildings, Edinburgh EH9 3JZ, UK \\ Received 2015 June 22; accepted 2015 December 4; published 2016 January 22
}

\begin{abstract}
The couplings between supermassive black hole binaries (SMBHBs) and their environments within galactic nuclei have been well studied as part of the search for solutions to the final parsec problem. The scattering of stars by the binary or the interaction with a circumbinary disk may efficiently drive the system to sub-parsec separations, allowing the binary to enter a regime where the emission of gravitational waves can drive it to merger within a Hubble time. However, these interactions can also affect the orbital parameters of the binary. In particular, they may drive an increase in binary eccentricity which survives until the system's gravitational-wave (GW) signal enters the pulsar-timing array (PTA) band. Therefore, if we can measure the eccentricity from observed signals, we can potentially deduce some of the properties of the binary environment. To this end, we build on previous techniques to present a general Bayesian pipeline with which we can detect and estimate the parameters of an eccentric SMBHB system with PTAs. Additionally, we generalize the PTA $\mathcal{F}_{\mathrm{e}}$-statistic to eccentric systems, and show that both this statistic and the Bayesian pipeline are robust when studying circular or arbitrarily eccentric systems. We explore how eccentricity influences the detection prospects of single GW sources, as well as the detection penalty incurred by employing a circular waveform template to search for eccentric signals, and conclude by identifying important avenues for future study.
\end{abstract}

Key words: gravitational waves - methods: data analysis - pulsars: general

\section{INTRODUCTION}

The observation of extremely compact objects-black holes (BHs), neutron stars (NSs), and white dwarfs-and the development of a thorough theoretical understanding of their nature has been one of the triumphs of modern astrophysics (Misner et al. 1973; Chandrasekhar 1983, p. 663; Thorne 1987, pp. 330-458), but there is still much that we do not understand about these exotic objects. The combination of electromagnetic observations with future detections of gravitational-wave $(\mathrm{GW})$ signals will provide key insights into the nature of compact objects and the role they play in some of the most energetic events in the universe: gamma-ray bursts, active galactic nuclei, quasars, etc. (Soltan 1982; Kormendy \& Richstone 1995; Magorrian et al. 1998; Janka et al. 1999; Gebhardt et al. 2000; Hughes \& Blandford 2003; Peterson et al. 2004; Lee \& Ramirez-Ruiz 2007; Hughes 2009; Metzger \& Berger 2012; Berger 2013; Berger et al. 2013; Piran et al. 2013; Tanvir et al. 2013). Several large-scale collaborations are working to inaugurate the new field of GW astronomy by targeting a wide variety of potential GW sources. These range from the mergers of supermassive black hole binaries (SMBHBs), which may be used by pulsar timing arrays (PTAs) to probe the innermost regions of merging galaxies, to the coalescence of NS binaries and stellar mass BHs, which encode important information about stellar evolution, galactic nuclei and globular clusters, and are the principle targets for ground-based GW detectors.

In this article, we will focus on a particular type of source that is being targeted by PTAs (Foster \& Backer 1990). PTAs aim to observe GWs in the nanohertz frequency band via the accurate timing of millisecond pulsars. There are three major
PTA collaborations - the European PTA, (Kramer \& Champion 2013), the North American Nanohertz Observatory for Gravitational-waves (NANOGrav, McLaughlin 2013) and the Parkes PTA (Hobbs 2013) in Australia. These three collaborations also aim to cooperate as the International PTA (IPTA, Manchester \& IPTA 2013).

The sources of interest in this work are individual SMBHBs during their early inspiral evolution (Rajagopal \& Romani 1995; Jaffe \& Backer 2003; Wyithe \& Loeb 2003; Sesana et al. 2009; Sesana \& Vecchio 2010a). Given the nature of these systems, i.e., large orbital separations and small local velocity of the binary components, we can take the compact objects as point-particles without internal dynamics and model the orbital evolution of the system using a post-Newtonian expansion (Peters \& Mathews 1963; Barack \& Cutler 2004; Sesana \& Vecchio 2010b). Furthermore, these events will be observed at large orbital separations, where the orbital evolution may be more strongly influenced by dynamical interactions with the astrophysical environment rather than GW emission. Hence, the circularizing influence of the latter may be lessened, allowing for quite large orbital eccentricities at the time of detection.

There are several mechanisms that could drive the eccentricity evolution of a SMBHB. For instance, at subparsec scales a binary formed by a galactic merger may be embedded in a dense stellar environment. As discussed in Sesana et al. (2008), if one assumes an isotropic stellar distribution, the interaction of a star and a SMBHB with semimajor axis $a$ can have two possible outcomes. Denoting the semimajor axis of the binary formed by the star and the SMBHB by $a_{\star}$, encounters with stars with $a_{\star} \lesssim a$ tend to circularize the orbit, whereas those with stars with $a_{\star} \gtrsim a$ tend 
to increase the eccentricity of the binary. In non-isotropic environments, co-rotation of the stellar distribution tends to circularize the binary. Counter-rotating stars tend to extract angular momentum from the SMBHB, causing the eccentricity to grow (Sesana et al. 2011). Several issues still remain to be explored regarding the evolution of SMBHBs at sub-parsec scales in dense stellar environments, but most models seem to favor a growth in orbital eccentricities before these systems enter the frequency band of PTAs (Sesana 2010; Roedig \& Sesana 2012).

Aside from interactions with stars, the dynamical evolution of a SMBHB at sub-parsec orbital separations can also be influenced by the redistribution of energy and angular momentum between the binary and a self-gravitating disk. Consider a gaseous disk co-rotating with a binary, and define $\lambda \equiv R_{t} / a$, where $R_{t}$ is the distance of the strongest torque on the binary as measured from the center of mass, and $a$ is the semimajor axis of the binary. Detailed numerical simulations suggest that the evolution of the orbital eccentricity of a SMBHB embedded in a circumbinary disk is independent of the mass-ratio of the system, but depends sensitively on the location of the inner rim of the disk, $\lambda$, with respect to the binary's center of mass. For $2<\lambda<2.5$, it is expected that binaries will converge to a critical eccentricity value $0.55<e_{*}<0.79$. Binaries with initial eccentricities $e>e_{*}$ will undergo a steady decrease in eccentricity, whereas binaries with $e<e_{*}$ will experience the opposite behavior. The larger the separation between the rim of the disk and the center of mass of the binary, the longer the system will take to attain $e_{*}$ (Roedig et al. 2011).

Taking into account these considerations, and the fact that uncertainties about the environments of binaries in realistic galaxy mergers make binary eccentricity a legitimate possibility, we recently introduced a theoretical framework to explore in detail the effect of eccentricity for source detection of potential PTA sources (Huerta et al. 2015). We now extend that analysis by introducing novel, accurate and efficient pipelines that shed light on the accuracy with which the astrophysical parameters of individually resolved eccentric SMBHBs can be reconstructed. This analysis explores the impact of eccentricity both in terms of source detection and parameter estimation, and presents new statistics to facilitate the analysis. Our approach builds on previous Bayesian (Ellis 2013; Taylor et al. 2014) and frequentist (Babak \& Sesana 2012; Ellis et al. 2012) statistics which have assumed circular gravitational waveform models, and unlike recent studies (Zhu et al. 2015), can recover all binary characteristics in addition to providing detection statistics. The latter study defined a frequentist statistic in terms of a harmonic sum over the lowest two harmonics, whereas we proceed from the full GW strain model of an eccentric binary, producing analytic signal models for Bayesian PTA singlesource GW searches, and a well-motivated frequentist statistic which fully generalizes that of Babak \& Sesana (2012) and Ellis et al. (2012).

This article is laid out as follows. In Section 2 we briefly review the orbital trajectories of eccentric binary systems, and how we can analytically solve for the orbital phase at a given time. This is followed in Section 3 by a description of the eccentric gravitational waveforms we use, and in Section 4 by our model of the perturbations these GWs induce in the times of arrival of radio signals from pulsars. The details of our analysis are provided in Section 5, followed by the results of
Bayesian and frequentist signal recoveries from simulated data sets in Section 6. In Section 7, we discuss the likely impact of several assumptions that we have made which should be explored further in future studies. We finish with concluding remarks in Section 8. In the following we adopt units such that $G=c=1$.

\section{ECCENTRIC BINARY ORBITS}

We briefly review the Kepler problem and present the general approach to analytically solve for the orbit of an eccentric binary, reiterating some of the notation and formalism of Yunes et al. (2009), and referring the reader to Goldstein (1950) for a more complete discussion.

We consider a binary system with component masses $m_{1}$ and $m_{2}$, total mass $M$, and a reduced mass $\mu=m_{1} m_{2} /\left(m_{1}+m_{2}\right)$. The separation vector joining the components is defined in terms of the component position vectors by $\boldsymbol{r}=\boldsymbol{r}_{1}-\boldsymbol{r}_{2}$, such that $\boldsymbol{r}_{1}=m_{2} \boldsymbol{r} / M$ and $\boldsymbol{r}_{2}=-m_{1} \boldsymbol{r} / M$. Using $(r=|\boldsymbol{r}|, \Phi)$ to denote plane polar coordinates for the position of one member of the binary with respect to the other, the Newtonian Keplerian orbital trajectories of two point particles in an eccentric binary system are described by

$$
\begin{gathered}
r=a(1-e \cos u), \\
\omega\left(t-t_{0}\right)=l=u-e \sin u \\
\Phi-\Phi_{0}=v \equiv 2 \arctan \left[\left(\frac{1+e}{1-e}\right)^{1 / 2} \tan \frac{u}{2}\right]
\end{gathered}
$$

where $a$ is the semimajor axis of the orbit, and $0 \leqslant e<1$ is the eccentricity (of a bound orbit). The eccentric anomaly, $u$, is an auxiliary variable with which to parametrize the radial and phase coordinates. Given the average angular frequency (or mean motion; $\omega=2 \pi / T$, where $T$ is the orbital period) and eccentricity of the orbit, we can solve the transcendental Equation (2) for $u$ at a given time $t$, where $l=2 \pi\left(t-t_{0}\right) / T$ is denoted as the mean anomaly. The eccentric anomaly can then be plugged into Equations (1) and (3) to give the separation and orbital phase (or true anomaly; $\Phi-\Phi_{0}$ ) at any point along the orbital trajectory. If we assume that time and phase are measured from the moment of periapsis, then the constants of integration $t_{0}$ and $\Phi_{0}$ can be set to zero. All of these angular quantities are shown diagrammatically for an example orbital ellipse in Figure 1.

The flux of energy and angular-momentum carried away from the system by GWs depend on the eccentricity and the Keplerian mean orbital frequency, $F$. Once the binary evolution is driven solely by GW emission, these co-evolve as (Peters 1964)

$$
\frac{F(e)}{F\left(e_{0}\right)}=\left(\frac{\sigma\left(e_{0}\right)}{\sigma(e)}\right)^{3 / 2},
$$

where

$$
\sigma(e)=\frac{e^{12 / 19}}{1-e^{2}}\left[1+\frac{121}{304} e^{2}\right]^{870 / 2299},
$$

and $e_{0}$ is defined as the eccentricity of the system at some earlier reference epoch of the binary evolution.

The frequency $F$ can be regarded as the instantaneous mean orbital frequency. For GW-dominated orbital evolution, it coevolves with the eccentricity according to the coupled 


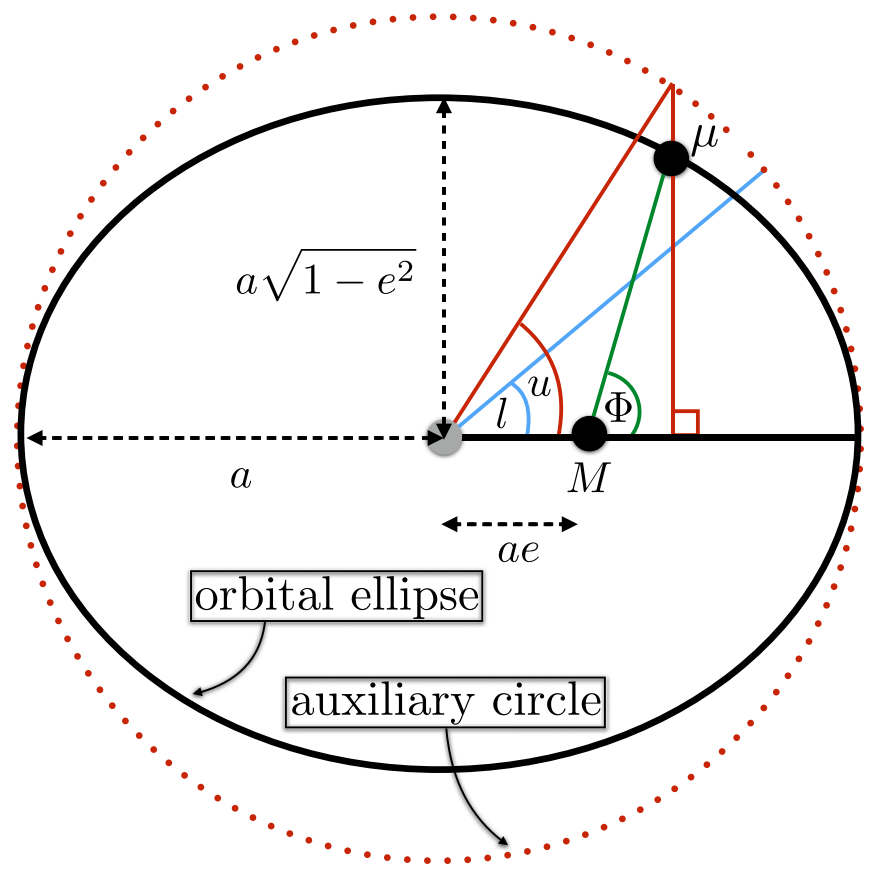

Figure 1. A diagram illustrating the relationship between the various angular elements in a binary system with orbital eccentricity $e$, reduced mass $\mu$, and total mass $M$. The semimajor and semiminor axes are $a$ and $a \sqrt{1-e^{2}}$, respectively. If we measure the angles from the moment of periapsis, then $\Phi$ is the true anomaly, $l$ is the mean anomaly, and $u$ is the eccentric anomaly. The auxiliary circle has a radius equal to the orbital semimajor axis.

differential equations (Peters 1964)

$$
\begin{aligned}
\frac{d F}{d t} & =\frac{48}{5 \pi \mathcal{M}^{2}}(2 \pi \mathcal{M} F)^{11 / 3} \frac{1+\frac{73}{24} e^{2}+\frac{37}{96} e^{4}}{\left(1-e^{2}\right)^{7 / 2}}, \\
\frac{d e}{d t} & =-\frac{304}{15 \mathcal{M}}(2 \pi \mathcal{M} F)^{8 / 3} e \frac{1+\frac{121}{304} e^{2}}{\left(1-e^{2}\right)^{5 / 2}},
\end{aligned}
$$

where $\mathcal{M}=\left(m_{1} m_{2}\right)^{3 / 5} /\left(m_{1}+m_{2}\right)^{1 / 5}$ is the binary chirp mass.

Gravitational waveform templates describing the emission from inspiraling binary systems depend on trigonometric functions of the orbital phase. For circular systems the relationship between orbital frequency, time, and phase is simple: we have $\Phi=2 \pi \int F(t) d t$, where $F(t)$ is the Keplerian orbital-frequency (half of the dominant quadrupole $\mathrm{GW}$ frequency) which evolves according to Equation (6) with $e=0$. However the situation is rather more complicated for eccentric systems. The phase is related via an arctangent to the eccentric anomaly, which is then related to the mean anomaly (and thus the mean angular frequency $\omega=2 \pi F$ ) via a transcendental equation. The so-called Kepler problem refers to the historical difficulty in finding solutions to the transcendental equation in Equation (2) and thus being able to express the orbital phase in terms of the mean anomaly. We do so using the well known Fourier analysis of the Kepler problem. For full details of the calculation see Watson (1995). Using elementary properties of elliptic curves and Bessel functions, the results are

$$
\cos \Phi=-e+\frac{2}{e}\left(1-e^{2}\right) \sum_{n=1}^{\infty} J_{n}(n e) \cos (n l),
$$

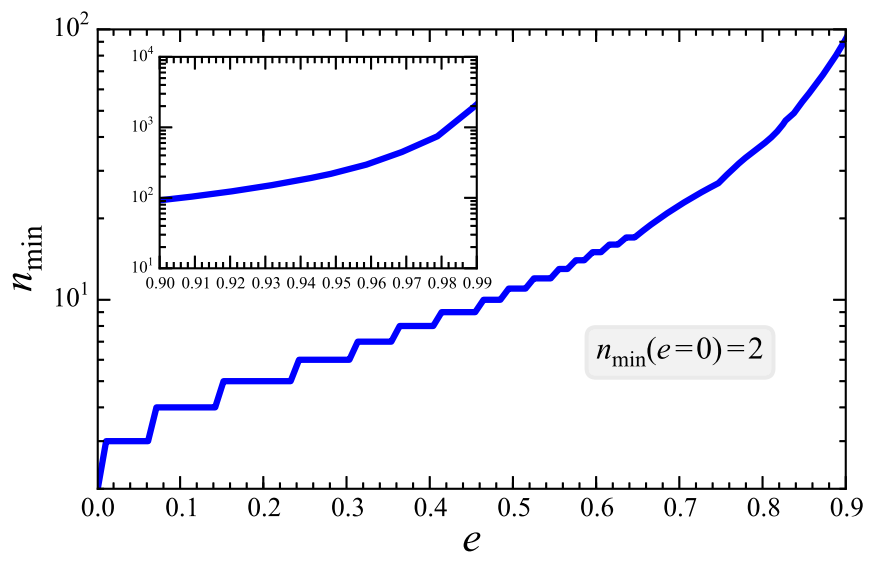

Figure 2. The minimum number of harmonics required for the Fourier solution of $\cos \Phi$ as a function of $l$ (mean anomaly) to maintain accuracy with the numerical solution. We demand that the overlap of the Fourier solution and numerical solution, as determined by the normalized scalar product of the two solution vectors, is $>99.999 \%$ over $2 \pi$ of mean anomaly.

$$
\sin \Phi=\left(1-e^{2}\right)^{1 / 2} \sum_{n=1}^{\infty}\left[J_{n-1}(n e)-J_{n+1}(n e)\right] \sin (n l) .
$$

With these trigonometric functions of the orbital phase, we can now construct gravitational waveforms for eccentric inspiraling binary systems in terms of the mean orbital frequency. Equations (7) and (8) can be immediately used to construct these waveforms. However, by setting a required tolerance on the accuracy of $\sin \Phi$ and $\cos \Phi$ for a given eccentricity, we can truncate the infinite summations (Pierro et al. 2001; Yunes et al. 2009) to accelerate calculations. We investigate the minimum number of terms required for the Fourier series expansion of $\cos \Phi$ in Equation (7) to maintain accuracy with the exact numerical solution of Equations (1)(3), by demanding that the error in the two solutions (determined by the normalized scalar product between the two solution vectors) is less than $0.001 \%$ over $2 \pi$ of meananomaly. The results are shown in Figure 2, where we see that $\lesssim 100$ terms in the summation are necessary to maintain accuracy up to $e=0.9$, however the required number of terms dramatically increases beyond 0.9 , exceeding $10^{3}$ at $e=0.99$.

Although systems with high residual eccentricity $(>0.9)$ in the sub-parsec GW inspiral regime may exist, they are by no means expected to be common. Unequal mass systems with $q \lesssim 0.25$ may retain $e>0.9$ into the PTA band (Khan et al. 2012), but we are unlikely to detect their weaker GW emission, so we focus here on the more probable case of a detectable signal from a comparable mass binary. Comparable mass binaries in isolated galaxy simulations exhibit $e<0.95$ when they transition from stellar hardening to GW-dominated evolution, although preliminary merger simulations can produce binaries with larger eccentricity (Vasiliev et al. 2015). However, since GW emission is well known to decrease eccentricity (Peters \& Mathews 1963), we believe the assumption that most detectable systems will likely have $e<0.9$ in the PTA band is astrophysically well-motivated, in addition to simplifying things computationally. Ultimately, the range of orbital separations at which the transition between stellar hardening and radiation-reaction occurs in real galaxies is a matter of debate (along with the range of possible binary eccentricities in the PTA band), and may only be resolved with pulsar-timing measurements. Hence, in the following we 
restrict our attention to systems with eccentricity below 0.9 , in which regime highly accurate waveforms require the inclusion of fewer than 100 Fourier terms.

\section{ECCENTRIC TIME-DOMAIN WAVEFORMS}

In the transverse-traceless gauge the GW-tensor can be written as a linear superposition of "plus" and "cross" polarization modes, with associated polarization-amplitudes, $h_{\{+, \times\}}$, and basis-tensors, $e_{a b}^{\{+, \times\}}(\hat{\Omega})$, such that

$$
h_{a b}(t, \hat{\Omega})=h_{+}(t) e_{a b}^{+}(\hat{\Omega})+h_{\times}(t) e_{a b}^{\times}(\hat{\Omega}),
$$

where $\hat{\Omega}$ is defined as the direction of GW propagation.

We employ the Peters-Mathews waveforms (Peters \& Mathews 1963) given by Barack \& Cutler (2004), which make use of the Fourier analysis of the Kepler problem to give the following analytic expressions for $h_{+}$and $h_{\times}$:

$$
\begin{aligned}
h_{+}(t)= & \sum_{n}-\left(1+\cos ^{2} \iota\right)\left[a_{n} \cos (2 \gamma)-b_{n} \sin (2 \gamma)\right] \\
& +\left(1-\cos ^{2} \iota\right) c_{n}, \\
h_{\times}(t)= & \sum_{n} 2 \cos \iota\left[b_{n} \cos (2 \gamma)+a_{n} \sin (2 \gamma)\right],
\end{aligned}
$$

where

$$
\begin{aligned}
a_{n}= & -n \zeta \omega^{2 / 3}\left[J_{n-2}(n e)-2 e J_{n-1}(n e)+(2 / n) J_{n}(n e)\right. \\
& \left.+2 e J_{n+1}(n e)-J_{n+2}(n e)\right] \cos [n l(t)], \\
b_{n}= & -n \zeta \omega^{2 / 3} \sqrt{1-e^{2}}\left[J_{n-2}(n e)\right. \\
& \left.-2 J_{n}(n e)+J_{n+2}(n e)\right] \sin [n l(t)], \\
c_{n}= & 2 \zeta \omega^{2 / 3} J_{n}(n e) \cos [n l(t)] .
\end{aligned}
$$

The amplitude parameter is defined as $\zeta=\mathcal{M}^{5 / 3} / D_{\mathrm{L}}$, where $D_{\mathrm{L}}$ is the luminosity distance of the binary, and $\omega=2 \pi F$. The mean anomaly is $l(t)=l_{0}+2 \pi \int_{t_{0}}^{t} F\left(t^{\prime}\right) d t^{\prime}$ (where $l_{0}$ is the mean anomaly at $\left.t_{0}\right) ; \gamma$ is an azimuthal angle measuring the direction of pericenter with respect to $\hat{x} \equiv(\hat{\Omega}+\hat{L} \cos \iota) / \sqrt{1-\cos ^{2} \iota}$; and $\iota$ is the binary orbital inclination angle, defined by $\cos \iota=-\hat{L} \cdot \hat{\Omega}$. In the following, $F$ and $\mathcal{M}$ refer to the observed redshifted values, such that $F_{\mathrm{r}}=F(1+z)$ and $\mathcal{M}_{\mathrm{r}}=\mathcal{M} /(1+z)$, where $F_{\mathrm{r}}$ and $\mathcal{M}_{\mathrm{r}}$ are rest frame values, and $z$ is the cosmological redshift of the binary.

An important feature to emphasize here is that eccentric binaries do not radiate monochromatic GWs, but rather emit a spectrum of frequencies which are harmonics of the mean orbital frequency. Given that $J_{0}(0)=1$ and $J_{n>0}(0)=0$, it is immediately obvious from Equations (10) and (11) that $e=0$ waveforms will only include the $n=2$ harmonic of the binary's mean orbital frequency. This is the usual result that the $\mathrm{GW}$ frequency of emission from circular binaries is twice the orbital frequency.

To construct the polarization basis tensors, we define a righthanded basis triad in terms of $\{\hat{n}, \hat{p}, \hat{q}\}$, where $\hat{n}=-\hat{\Omega}$, $\hat{p}=(\hat{n} \times \hat{L}) /|\hat{n} \times \hat{L}|$ and $\hat{q}=\hat{p} \times \hat{n}$. The vectors comprising the basis triad are explicitly

$$
\begin{gathered}
\hat{n}=(\sin \theta \cos \phi, \sin \theta \sin \phi, \cos \theta), \\
\hat{p}=(\cos \psi \cos \theta \cos \phi-\sin \psi \sin \phi, \\
\cos \psi \cos \theta \sin \phi+\sin \psi \cos \phi,-\cos \psi \sin \theta),
\end{gathered}
$$

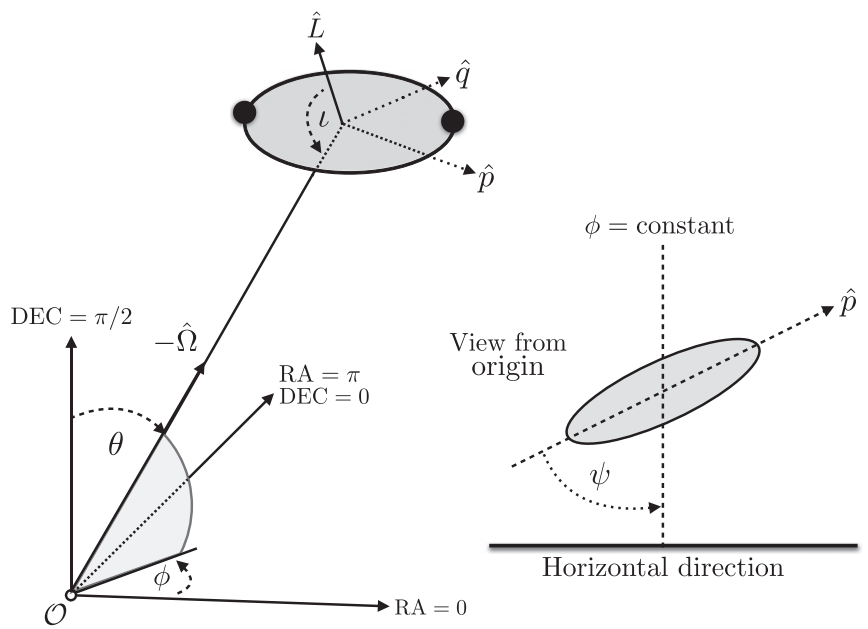

Figure 3. A diagram illustrating the geometry of an eccentric SMBHB with respect to the angles of our coordinate system. The unit vector pointing to the binary is $\hat{n}=-\hat{\Omega}$, with spherical-polar coordinates $\{\theta=\pi / 2-$ decl., $\phi=\mathrm{R}$. A. $\}$. The binary orbital inclination angle is defined by $\cos \iota=\hat{L} \cdot \hat{n}$, where $\hat{L}$ is a unit vector pointing along the binary's orbital angular momentum. The GW polarization basis tensors are defined in the plane transverse to the direction of propagation, in terms of the unit vectors $\hat{p}=(\hat{n} \times \hat{L}) /|\hat{n} \times \hat{L}|$ and $\hat{q}=\hat{p} \times \hat{n}$, where $\{\hat{n}, \hat{p}, \hat{q}\}$ define a right-handed basis triad. The vector $\hat{p}$ lies along the major axis of the projected ellipse as seen from the origin of the coordinate system. The GW polarization angle $\psi$ is defined as the angle between $\hat{p}$ and the line of constant azimuth. This diagram is a modified version of Figure 1 in Apostolatos et al. (1994).

$$
\begin{aligned}
\hat{q}= & (\sin \psi \cos \theta \cos \phi+\cos \psi \sin \phi, \\
& \sin \psi \cos \theta \sin \phi-\cos \psi \cos \phi,-\sin \psi \sin \theta),
\end{aligned}
$$

where $(\theta, \phi)=(\pi / 2-$ decl., R.A. $)$ denotes the sky-location of the binary in spherical polar coordinates, and $\psi$ corresponds to the angle between $\hat{p}$ and the line of constant azimuth when the orbit is viewed from the origin of our coordinate system. These angles are shown diagrammatically in Figure 3 . The vectors $\hat{p}$ and $\hat{q}$ lie in the plane that is transverse to the direction of GW propagation, and are used to construct basis tensors as follows:

$$
\begin{aligned}
& e_{a b}^{+}=\hat{p}_{a} \hat{p}_{b}-\hat{q}_{a} \hat{q}_{b}, \\
& e_{a b}^{\times}=\hat{p}_{a} \hat{q}_{b}+\hat{q}_{a} \hat{p}_{b} .
\end{aligned}
$$

\section{PULSAR TIMING RESIDUALS INDUCED BY AN ECCENTRIC BINARY}

As a GW transits across the line of sight between a pulsar and the Earth, it creates a perturbation in the spacetime metric which causes a change in the proper separation between the Earth and the pulsar. This in turn leads to a shift in the perceived pulsar rotational frequency. The fractional frequency shift of a signal from a pulsar in the direction of unit vector $\hat{u}$, induced by the passage of a single GW propagating in the direction of $\hat{\Omega}$ is (Anholm et al. 2009; Book \& Flanagan 2011)

$$
z(t, \Omega)=\frac{1}{2} \frac{\hat{u}^{a} \hat{u}^{b}}{1+\hat{\Omega} \cdot \hat{u}} \Delta h_{a b}(t, \Omega),
$$

where $\Delta h_{a b} \equiv h_{a b}\left(t_{\mathrm{e}}, \hat{\Omega}\right)-h_{a b}\left(t_{\mathrm{p}}, \hat{\Omega}\right)$ is the difference in the metric perturbation evaluated at time $t_{\mathrm{e}}$ when the GW passed the solar system barycenter and time $t_{\mathrm{p}}$ when the GW passed the pulsar. From simple geometrical arguments, we can write 
$t_{\mathrm{p}}=t_{\mathrm{e}}-L(1+\hat{\Omega} \cdot \hat{u})$, where $L$ is the distance to the pulsar. The integrated effect of this GW-induced redshift over the total observing time of the pulsar leads to an offset between the expected and the observed pulse TOA:

$$
s(t)=\int_{0}^{t} z\left(t^{\prime}\right) d t^{\prime} .
$$

The expected pulse TOA is computed from a deterministic timing model which characterizes a pulsar's astrometric and spin properties. This model is refined over many observations to give an accurate prediction of the pulse arrival times. The difference between the measured TOAs and those predicted by the best-fit deterministic timing-model are the timing residuals. In addition to any GW signals, these residuals encode the influence of noise processes and all unmodeled phenomena which affect pulsar TOAs. The pulsar timing residuals induced by a single GW source can be written as

$$
s(t, \hat{\Omega})=F^{+}(\hat{\Omega}) \Delta s_{+}(t)+F^{\times}(\hat{\Omega}) \Delta s_{\times}(t),
$$

where $\quad A=\{+, \times\}, \quad \Delta s_{A}(t)=s_{A}\left(t_{\mathrm{e}}\right)-s_{A}\left(t_{\mathrm{p}}\right), \quad$ with $s_{A}(t)=\int_{0}^{t} h_{A}\left(t^{\prime}\right) d t^{\prime}$, and $F^{A}(\hat{\Omega})$ are antenna pattern response functions encoding the geometrical sensitivity of a particular pulsar to a propagating $\mathrm{GW}$, defined as

$$
F^{A}(\hat{\Omega}) \equiv \frac{1}{2} \frac{\hat{u}^{a} \hat{u}^{b}}{1+\hat{\Omega} \cdot \hat{u}} e_{a b}^{A}(\hat{\Omega}),
$$

and corresponding to the contraction of the pulsar-timing impulse response function with the $\mathrm{GW}$ polarization basis tensors.

The form of $s_{A}(t)$ can be computed analytically by assuming that the binary's mean orbital frequency and eccentricity remain constant over the total timespan of our observations of a given pulsar. More specifically, we must assume no binary evolution over the Earth term timing baseline, $\left[t_{\mathrm{e}}, t_{\mathrm{e}}+T\right]$, and also the corresponding timing baseline of the pulsar term, $\left[t_{\mathrm{e}}-L(1+\hat{\Omega} \cdot \hat{u}), t_{\mathrm{e}}+T-L(1+\hat{\Omega} \cdot \hat{u})\right]$, where $T$ is $\mathcal{O}\left(10\right.$ years). ${ }^{6}$ Therefore, time only appears in the definition of the mean anomaly as a linear parameter, such that $l(t)=l_{0}+2 \pi \int_{t_{0}}^{t} F\left(t^{\prime}\right) d t^{\prime}=l_{0}+2 \pi F\left(t-t_{0}\right), \quad$ which allows $\cos [n l(t)]$ and $\sin [n l(t)]$ in Equation (11) to be trivially integrated to give the plus/cross residuals:

$$
\begin{aligned}
s_{+}(t)= & \sum_{n}-\left(1+\cos ^{2} \iota\right)\left[a_{n} \cos (2 \gamma)-b_{n} \sin (2 \gamma)\right] \\
& +\left(1-\cos ^{2} \iota\right) c_{n}, \\
s_{\times}(t)= & \sum_{n} 2 \cos \iota\left[b_{n} \cos (2 \gamma)+a_{n} \sin (2 \gamma)\right],
\end{aligned}
$$

\footnotetext{
6 The binary's mean orbital frequency and eccentricity do evolve nonnegligibly over the light travel time between the Earth and the pulsar, $\mathcal{O}(1000$ years $)-\mathcal{O}(10000$ years $)$. This effect is easily included in our signal model, however in the rest of this paper we consider only the Earth term.
}

where

$$
\begin{aligned}
a_{n}= & -\zeta \omega^{-1 / 3}\left[J_{n-2}(n e)-2 e J_{n-1}(n e)+(2 / n) J_{n}(n e)\right. \\
& \left.+2 e J_{n+1}(n e)-J_{n+2}(n e)\right] \sin [n l(t)] \\
= & \zeta \omega^{-1 / 3} x_{a_{n}} \sin [n l(t)], \\
b_{n}= & \zeta \omega^{-1 / 3} \sqrt{1-e^{2}}\left[J_{n-2}(n e)-2 J_{n}(n e)\right. \\
& \left.+J_{n+2}(n e)\right] \cos [n l(t)] \\
= & \zeta \omega^{-1 / 3} x_{b_{n}} \cos [n l(t)], \\
c_{n}= & (2 / n) \zeta \omega^{-1 / 3} J_{n}(n e) \sin [n l(t)] \\
= & \zeta \omega^{-1 / 3} x_{c_{n}} \sin [n l(t)],
\end{aligned}
$$

and the quantities $\left\{x_{a_{n}}, x_{b_{n}}, x_{c_{n}}\right\}$ are defined for later convenience.

We can now analyze the harmonic content of the variance of the residuals from both plus and cross polarizations, which is computed over one period of binary elliptical motion $(l=\{0,2 \pi\})$ and over $\cos \iota, \gamma$. Clearly averaging over a single (or any non-zero integer) period of orbital motion is only an approximation, since our pulsar-timing observations are highly unlikely to span an integer number of orbital periods or GW cycles. Nevertheless we carry out this calculation since it illuminates certain features of the harmonic content of the $\mathrm{GW}$ signal from eccentric SMBHBs. We employ the following relations when averaging over the mean anomaly:

$$
\begin{aligned}
& \int_{0}^{2 \pi} d l \sin (n l) \cos \left(n^{\prime} l\right)=0, \forall n, n^{\prime}, \\
& \int_{0}^{2 \pi} d l \sin (n l) \sin \left(n^{\prime} l\right)= \begin{cases}0, & \text { if } n \neq n^{\prime}, \\
\pi, & \text { if } n=n^{\prime},\end{cases}
\end{aligned}
$$

where $n, n^{\prime} \geqslant 1$, and the last equation is also true for cosine functions. Given that the induced residuals are zero-mean over integers of the binary orbital period, the resulting variance of the residuals is

$$
\left\langle s_{A}^{2}\right\rangle=\zeta^{2} \omega^{-2 / 3} \sum_{n}\left\langle s_{A}^{2}\right\rangle_{n},
$$

where

$$
\begin{aligned}
\left\langle s_{+}^{2}\right\rangle_{n} & =\frac{7}{15}\left(x_{a_{n}}^{2}+x_{b_{n}}^{2}\right)+\frac{4}{15} x_{c_{n}}^{2}, \\
\left\langle s_{\times}^{2}\right\rangle_{n} & =\frac{1}{3}\left(x_{a_{n}}^{2}+x_{b_{n}}^{2}\right) .
\end{aligned}
$$

The value of $\left\langle s_{+}^{2}\right\rangle_{n}$ for several binary eccentricities is shown in the left panel of Figure 4. At each eccentricity, the contribution of each harmonic to the variance of the residuals is normalized with respect to the largest contribution. In the right panel of Figure 4 we show the fraction of the total variance of the plus-component timing residuals contributed by the dominant harmonic, which switches from $n=2$ in the $0 \leqslant e \lesssim 0.4$ range to $n=1$ beyond $e \sim 0.4$.

For the remainder of this paper we will present results from investigations with the Earth term of the GW-induced timing residuals. The signal model in Equation (21) is general, and can be used to compute both Earth and pulsar terms, modulo the assumption of binary non-evolution over typical pulsar timing baselines. However, including the pulsar term requires either precise knowledge of the individual pulsar distances, or the distances to be searched or marginalized over (Ellis 2013; Taylor et al. 2014). This search over distance brings its own 

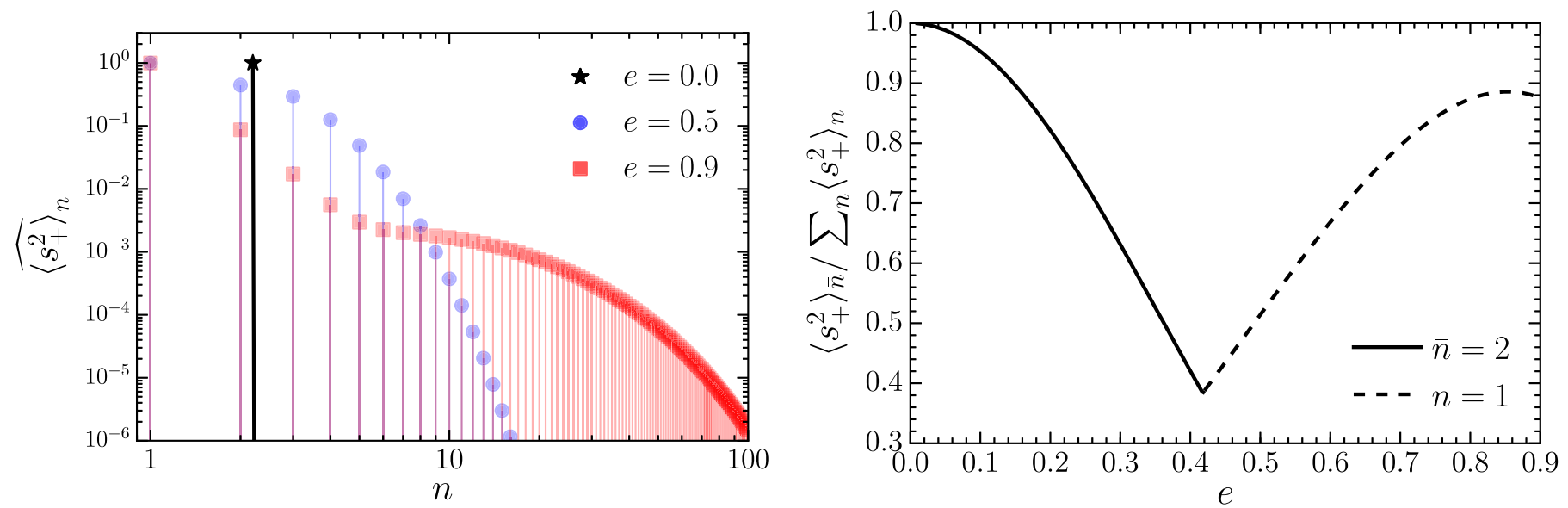

Figure 4. (Left): the contribution of each harmonic of the orbital frequency to the variance of the plus-component timing residuals. At each eccentricity we normalize the contributions from each harmonic with respect to the maximum contribution. The only contribution for circular binaries is from the second harmonic (black star and line, slightly offset from $n=2$ for ease of viewing). At higher eccentricities $(e=0.5,0.9)$ the contribution is spread into a spectrum of higher harmonics, but is dominated by the fundamental harmonic. (Right): the fraction of the total variance contributed by the dominant harmonic, $\bar{n}$, as a function of eccentricity. As in the left panel, $n$ labels the harmonic of the binary mean orbital frequency. In the range $0 \leqslant e \lesssim 0.4$ the second harmonic dominates, while beyond $e \sim 0.4$ the fundamental harmonic dominates the variance of the induced timing residuals.

challenges since the likelihood is highly sensitive to small changes in the sampled distance around the true value, and can lead to inefficient sampling. We defer considerations of the pulsar term to future work, but will briefly consider its influence in Section 7. Furthermore, for the most extreme combinations of binary mass, eccentricity, and orbital frequency, the system may exhibit frequency chirping and orbital circularization during typical pulsar-timing observation timespans, rendering the assumption of non-evolution invalid. We explore these issues in Section 7 amid suggestions for future directions.

Related to these two issues are the fact that in general we would also need to consider evolution of the direction of pericenter, $\dot{\gamma}$, and orbital plane precession from spin-orbit coupling. Evolution of the direction of pericenter can occur even for circular binary systems composed of non-spinning BHs, leading to phase shifts and recovery bias in the orbital frequency if it is not considered. However, as discussed in Sesana \& Vecchio (2010b), these factors can be safely ignored over typical PTA observation timespans. In Figure 5 we show exclusion regions in $\left\{M=\left(m_{1}+m_{2}\right), F, e\right\}$ parameter space, where pericenter direction evolution leads to a bias in the orbital frequency which is greater than the typical PTA frequency resolution of $1 / T$ for a 10 year observation timespan (Sesana \& Vecchio 2010b). The excluded regions correspond to systems with very high total mass and eccentricity, and orbital frequencies beyond the region of peak PTA sensitivity. Hence, we ignore this effect here and consider only $\{F, e\}$ evolution in Section 7, but information from these additional effects may allow the individual binary component masses, and possibly their spin, to be constrained (Mingarelli et al. 2012). Additionally, these effects are likely to be highly important when tracing the binary evolution back by thousands of years to the pulsar term.

\section{SIMULATED DATA SETS AND ANALYSIS}

For our proof-of-principle study of an eccentric single-source pipeline, we consider two types of PTA data sets. In our Type I

\footnotetext{
http://www.ipta4gw.org/?page_id=89
}

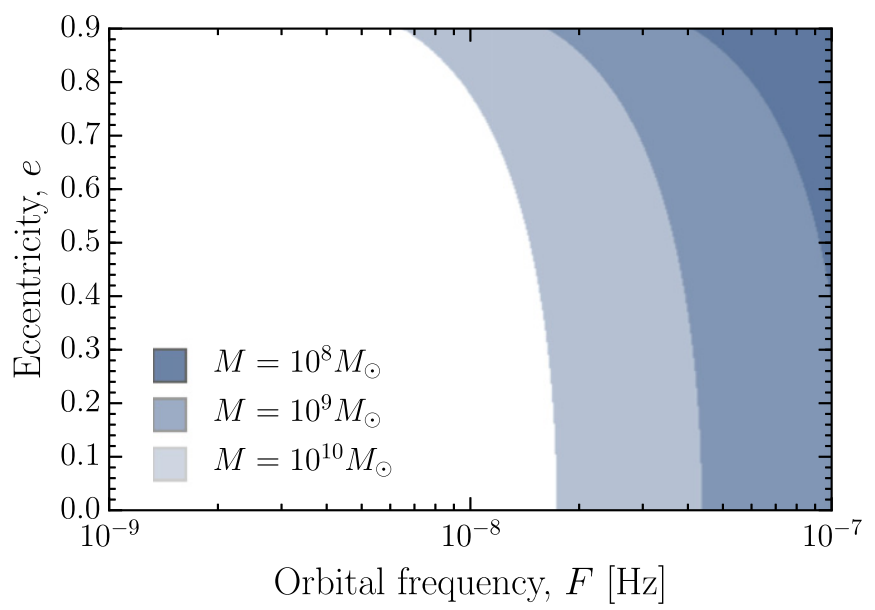

Figure 5. Exclusion regions in binary eccentricity and orbital frequency as a function of binary total mass, corresponding to parameter combinations where unmodeled evolution of binary pericenter direction causes a bias in orbital frequency recovery which could be resolved by 10 years of PTA observations, $\Delta f=1 / T=3.2 \mathrm{nHz}$.

array, we consider the 36 pulsars from the IPTA mock data challenge. ${ }^{7}$ They are timed to $100 \mathrm{~ns}$ precision over a timing baseline of 10 years, with observations carried out every four weeks. This array is obviously idealized, however the generalization to more realistic observing schedules and pulsar noise properties does not require modifications to our pipeline since it is constructed in the time-domain, and is shielded from Fourier domain spectral leakage caused by red timing noise or irregular sampling. The Bayesian pipeline can be trivially incorporated into a more general pipeline which simultaneously estimates pulsar noise properties and other stochastic signals. The Type I data sets will serve as the ideal observing scenario to test for any systematic errors in our signal construction which are separate from observing practicalities, and will also be used for brief analyses of the influence of binary eccentricity on circular- or eccentric-model signal-to-noise ratios (S/Ns).

To emulate more realistic observing schedules and pulsar noise properties, we also construct Type II data sets using the actual epochs of observation and noise properties of the 18 
pulsars that were used by the NANOGrav collaboration to place astrophysical constraints on the nanohertz GW background (Arzoumanian et al. 2015; NANOGrav Collaboration et al. 2015). These pulsars suffer from irregular sampling, different timing baselines (the longest is $\sim 9$ years), heteroscedastic TOA measurement errors, and, in some cases, intrinsic pulsar spin noise. These Type II arrays will be used for our Bayesian studies of the penalties arising from assuming a circular binary model when analyzing data having an eccentric signal, and also when estimating the precision with which current PTAs can estimate binary parameters.

We use the simulation routines within libstempo, ${ }^{8}$ a python wrapper for the pulsar-timing software package TEMPO2 (Edwards et al. 2006; Hobbs et al. 2006). For a fiducial source, we are only interested in sensible binary parameters which will illustrate the efficacy of the search pipeline. We follow Ellis (2013), Taylor et al. (2014) by considering a source with the following characteristics: $\left\{\mathcal{M}=10^{9} M_{\odot}, F=5 \mathrm{nHz}, \phi=0.95\right.$, $\left.\theta=2.17, \quad \iota=1.57, \quad l_{0}=0.99, \quad \psi=1.26, \quad \gamma=0.5\right\}$, and a luminosity distance scaled to meet a required optimal $\mathrm{S} / \mathrm{N}$. The definitions of optimal and matched-filtering $\mathrm{S} / \mathrm{N}$ follow from Finn (2001).

The binary parameter space is searched using a python wrapper (Buchner et al. 2014) to the nested sampling package MultiNest (Feroz \& Hobson 2008; Feroz et al. 2009, 2013), and we have cross-checked our results with a sampler utilizing advanced Markov chain Monte Carlo techniques. ${ }^{9}$ The product of these analyses are samples from the posterior probability distribution of the signal parameters space, allowing us to quantify the measurement precision of parameters based on the Bayesian credible regions, and also permitting model selection via computation of competing models' Bayesian evidence. The priors for the signal parameters are as follows: $\log _{10}\left(\mathcal{M} / M_{\odot}\right) \in U[7,10], \log _{10}\left(D_{\mathrm{L}} / \mathrm{Mpc}\right) \in U[0,4], \log _{10}$ $(F / \mathrm{Hz}) \in U[-9.3,-6.0], \quad e \in U[0,0.9], \quad \phi \in U[0,2 \pi]$, $\cos \theta \in U[-1,1], \cos \iota \in U[-1,1], \psi \in U[0, \pi], \gamma \in U[0, \pi]$, $l_{0} \in U[0,2 \pi]$.

Full details of Bayesian inference in the context of PTAs can be found in van Haasteren et al. (2009), van Haasteren \& Vallisneri (2014), NANOGrav Collaboration et al. (2015), and for details of how Bayesian searches for continuous GWs are carried out see Ellis (2013), Taylor et al. (2014).

\subsection{Eccentric $\mathcal{F}_{\mathrm{e}}$ Statistic}

Equation (21) provides the appropriate signal model to use when we wish to map out the posterior distribution of the entire signal parameter space, and also if we were to simultaneously search for continuous GW sources in addition to stochastic signal or noise processes. However, we can also construct a fixed-noise frequentist statistic for eccentric binary systems.

We wish to construct a form of the $\mathcal{F}_{\mathrm{e}}$ statistic (Babak \& Sesana 2012; Ellis et al. 2012) which can be applied to GW signals from binaries with arbitrary eccentricity. In practice, as in the rest of this paper, we only consider systems with $e \in[0,0.9]$. The $\mathcal{F}_{\mathrm{e}}$ statistic as it is constructed in Ellis et al. (2012) is a maximum-likelihood estimator of the source's skylocation and orbital frequency, and requires that the expression for the induced residuals be rearranged into a form which permits maximization of the likelihood-ratio over the

\footnotetext{
8 http://vallis.github.io/libstempo/

9 https://github.com/jellis18/PTMCMCSampler
}

coefficients of a set of time-dependent basis-functions. The likelihood-ratio, $\Lambda$, is defined as the ratio of the likelihood of the data in a model which includes a signal to the noise-only null hypothesis:

$$
\begin{aligned}
\ln \Lambda & =\ln \left[\frac{\mathcal{L}(\boldsymbol{s} \mid \delta \boldsymbol{t})}{\mathcal{L}(0 \mid \delta \boldsymbol{t})}\right] \\
& =(\delta \boldsymbol{t} \mid \boldsymbol{s})-\frac{1}{2}(\boldsymbol{s} \mid \boldsymbol{s}) .
\end{aligned}
$$

We extend the $\mathcal{F}_{\mathrm{e}}$ statistic by rewriting the Earth term residuals (in a single pulsar) given by Equations (21) and (22) as:

$$
s(t)=\sum_{i=1}^{6} w_{i} \mathcal{W}^{i}
$$

where,

$$
\begin{gathered}
w_{1}=\zeta\left[-\left(1+\cos ^{2} \iota\right) \cos (2 \gamma) \cos (2 \psi)\right. \\
+2 \cos \iota \sin (2 \gamma) \sin (2 \psi)], \\
w_{2}=\zeta\left[\left(1+\cos ^{2} \iota\right) \sin (2 \gamma) \cos (2 \psi)\right. \\
+2 \cos \iota \cos (2 \gamma) \sin (2 \psi)], \\
w_{3}=\zeta\left[\left(1-\cos ^{2} \iota\right) \cos (2 \psi)\right], \\
w_{4}=\zeta\left[\left(1+\cos ^{2} \iota\right) \cos (2 \gamma) \sin (2 \psi)\right. \\
+2 \cos \iota \sin (2 \gamma) \cos (2 \psi)], \\
w_{5}=\zeta\left[-\left(1+\cos ^{2} \iota\right) \sin (2 \gamma) \sin (2 \psi)\right. \\
\quad+2 \cos \iota \cos (2 \gamma) \cos (2 \psi)], \\
w_{6}=\zeta\left[-\left(1-\cos { }^{2} \iota\right) \sin (2 \psi)\right], \\
\mathcal{W}^{1}=\tilde{F}^{+}(\hat{\Omega}) \omega^{-1 / 3} \sum_{n} x_{a_{n}} \sin \left[n \omega\left(t-t_{0}\right)+n l_{0}\right], \\
\mathcal{W}^{2}=\tilde{F}^{+}(\hat{\Omega}) \omega^{-1 / 3} \sum_{n} x_{b_{n}} \cos \left[n \omega\left(t-t_{0}\right)+n l_{0}\right], \\
\mathcal{W}^{3}=\tilde{F}^{+}(\hat{\Omega}) \omega^{-1 / 3} \sum_{n} x_{c_{n}} \sin \left[n \omega\left(t-t_{0}\right)+n l_{0}\right], \\
\mathcal{W}^{4}=\tilde{F}^{\times}(\hat{\Omega}) \omega^{-1 / 3} \sum_{n} x_{a_{n}} \sin \left[n \omega\left(t-t_{0}\right)+n l_{0}\right], \\
\mathcal{W}^{5}=\tilde{F}^{\times}(\hat{\Omega}) \omega^{-1 / 3} \sum_{n} x_{b_{n}} \cos \left[n \omega\left(t-t_{0}\right)+n l_{0}\right], \\
\mathcal{W}^{6}=\tilde{F}^{\times}(\hat{\Omega}) \omega^{-1 / 3} \sum_{n} x_{c_{n}} \sin \left[n \omega\left(t-t_{0}\right)+n l_{0}\right],
\end{gathered}
$$

and we adapt the number of terms in these summations based on the binary eccentricity. This is the same adaptation as discussed in the previous section for the Bayesian analysis.

The antenna pattern functions $\tilde{F}^{A}(\hat{\Omega})$ are related to $F^{A}(\hat{\Omega})$ by

$$
\left(\begin{array}{c}
F^{+} \\
F^{\times}
\end{array}\right)=\left(\begin{array}{cc}
\cos (2 \psi) & -\sin (2 \psi) \\
\sin (2 \psi) & \cos (2 \psi)
\end{array}\right)\left(\begin{array}{c}
\tilde{F}^{+} \\
\tilde{F}^{\times}
\end{array}\right) .
$$

The coefficients $w_{i}$ are a function of extrinsic source parameters $\{\zeta, \iota, \psi, \gamma\}$, while the time-dependent basis-functions $\mathcal{W}^{i}$ are a function of intrinsic source parameters $\left\{F, \theta, \phi, e, l_{0}\right\}$.

Hence, the full PTA signal template can be written as:

$$
s(t)=\sum_{i=1}^{6} w_{i} \mathcal{W}^{i}(t),
$$


where,

$$
\mathcal{W}^{i}=\left[\begin{array}{c}
\mathcal{W}_{1}^{i}(t) \\
\mathcal{W}_{2}^{i}(t) \\
\vdots \\
\mathcal{W}_{N_{\mathrm{p}}}^{i}(t)
\end{array}\right],
$$

and $\mathcal{W}_{j}^{i}(t)$ denotes the quantity $\mathcal{W}^{i}$ defined by Equation (30) for pulsar $j$. Inserting Equation (32) into (27) and using Einstein summation convention, we have

$$
\ln \Lambda=w_{i} N^{i}-\frac{1}{2} M^{i j} w_{i} w_{j},
$$

where $N^{i}=\left(\delta \boldsymbol{t} \mid \boldsymbol{W}^{i}\right)$ and $M^{i j}=\left(\boldsymbol{W}^{i} \mid \boldsymbol{W}^{j}\right)$. By maximizing the log-likelihood ratio over the amplitude coefficients, $w_{i}$, we get their maximum-likelihood values:

$$
\hat{w}_{i}=M_{i j} N^{j},
$$

where $M_{i j}=\left(M^{i j}\right)^{-1} \cdot{ }^{10}$ Substituting these coefficients back into the expression for $\ln \Lambda$ gives the eccentric $\mathcal{F}_{\mathrm{e}}$ statistic:

$$
\mathcal{F}_{\mathrm{e}}=\frac{1}{2} N^{i} M_{i j} N^{j}
$$

The procedure to estimate the maximum likelihood values of all of the signal parameters is as follows:

1. We find the local maxima of the $\mathcal{F}_{\mathrm{e}}$ statistic in the space of intrinsic parameters via a straightforward function maximization, or we can map out the posterior distribution of the semi-maximized parameter space with a stochastic sampler, and determine the maximum likelihood point from the resulting chain.

2. The intrinsic parameters which maximize the $\mathcal{F}_{\mathrm{e}}$ statistic can be used to compute the quantities $M_{i j}$ and $N^{i}$, which are combined to determine the maximum likelihood coefficients, $w_{i}$, via Equation (35).

3. From these coefficients, we obtain a maximum likelihood estimate of the physical extrinsic parameters, as described below.

There are six $w_{i}$ parameters, but these are functions of only four physical extrinsic parameters and so not all combinations of $w_{i}$ 's correspond to physical systems. However, we can obtain extrinsic parameter estimates from estimates of the $w_{i}$ 's following (Cornish \& Porter 2007). We define

$$
\begin{aligned}
A_{+}= & \sqrt{\left(w_{1}+w_{5}\right)^{2}+\left(w_{2}-w_{4}\right)^{2}} \\
& +\sqrt{\left(w_{1}-w_{5}\right)^{2}+\left(w_{2}+w_{4}\right)^{2}}, \\
A_{\times}= & \sqrt{\left(w_{1}+w_{5}\right)^{2}+\left(w_{2}-w_{4}\right)^{2}} \\
& -\sqrt{\left(w_{1}-w_{5}\right)^{2}+\left(w_{2}+w_{4}\right)^{2}},
\end{aligned}
$$

and

$$
A=A_{+}+\sqrt{A_{+}^{2}-A_{\times}^{2}} .
$$

\footnotetext{
${ }^{10}$ Through practical experience we find that the inverted matrix has greater numerical stability at low eccentricity $(e \lesssim 0.05)$ when a Moore-Penrose pseudoinverse is used, with a typical singular value cutoff of $\sim 10^{-10}$.
}

By employing the quantities $\left\{A_{+}, A_{\times}, A\right\}$ we can map from $w_{i \in[1,6]}$ to $\{\zeta, \iota, \psi, \gamma\}$ with the following manipulations:

$$
\begin{aligned}
\zeta & =\frac{A}{4}, \\
\cos \iota & =-\frac{A_{\times}}{A}, \\
\tan (2 \psi) & =\frac{A_{\times} w_{1}-A_{+} w_{5}}{A_{\times} w_{4}+A_{+} w_{2}}, \\
\tan (2 \gamma) & =\frac{A_{\times} w_{1}-A_{+} w_{5}}{A_{+} w_{4}+A_{\times} w_{2}} .
\end{aligned}
$$

In the following we treat the eccentric $\mathcal{F}_{\mathrm{e}}$ statistic as a likelihood function and map out the posterior probability distribution of the semi-maximized signal parameter space.

\section{RESULTS}

\subsection{Efficacy of Pipelines}

We accelerate the generation of templates for the GWinduced residuals by making the number of waveform harmonics adapt based on the current proposed eccentricity. As discussed in Section 2, the number of harmonics to adequately describe a binary with $e=0.5$ is $\sim 10$, while for $e=0.9$ it is $\sim 100$. Adaptation of the number of harmonics avoids template generation being the main computational bottleneck in our pipeline.

As a first illustration of the efficacy of our pipelines, we inject $\mathrm{GW}$ signals with $\mathrm{S} / \mathrm{N}=20$ into noisy Type I data sets, and analyze the data with our Bayesian and frequentist statistics. We overlay the $95 \%$ envelope of Bayesian credible post-fit GW-induced residuals on top of the raw post-fit residuals from a single pulsar in our array. The results are shown for an $e=0$ and $e=0.5$ binary signal in Figure 6, where we see that the region of credible residuals (enclosed within red dashed lines) tracks the main features in the raw post-fit residuals, and correctly interprets high frequency behavior around MJD 55100 in the right panel $(e=0.5)$ as binary periapsis. In Figure 6 we also show the deviation of the recovered residuals from the true injected residuals, where the envelope of credible residuals encompasses the line of zero offset. This shows that, even in this high $\mathrm{S} / \mathrm{N}$ case, any systematic bias from the adaptation of the number of harmonics is very small, and our Bayesian pipeline is robustly recovering the signal characteristics.

We also use our samplers to map out the $\mathcal{F}_{\mathrm{e}}$ statistic distribution over the intrinsic parameter space. From the chain of sampled points we determine the maximum-likelihood intrinsic parameters, which are then used to construct $w_{i}$ via Equation (35). Having the maximized $w_{i}$ and corresponding $\mathcal{W}^{i}$, we now compute the maximum-likelihood timing residuals induced by the GWs from an eccentric binary. The results for the $e=0$ and $e=0.5$ binary signals are shown in Figure 6, where the maximum-likelihood GW-induced post-fit residuals are overlaid as black dashed lines on top of the raw post-fit residuals from pulsar $\mathrm{J} 0030+0451$, showing excellent tracking of the residual behavior and good agreement with the Bayesian recovery. Note that these maximum-likelihood residuals are offset by $+0.1 \mu$ s for ease of viewing. 

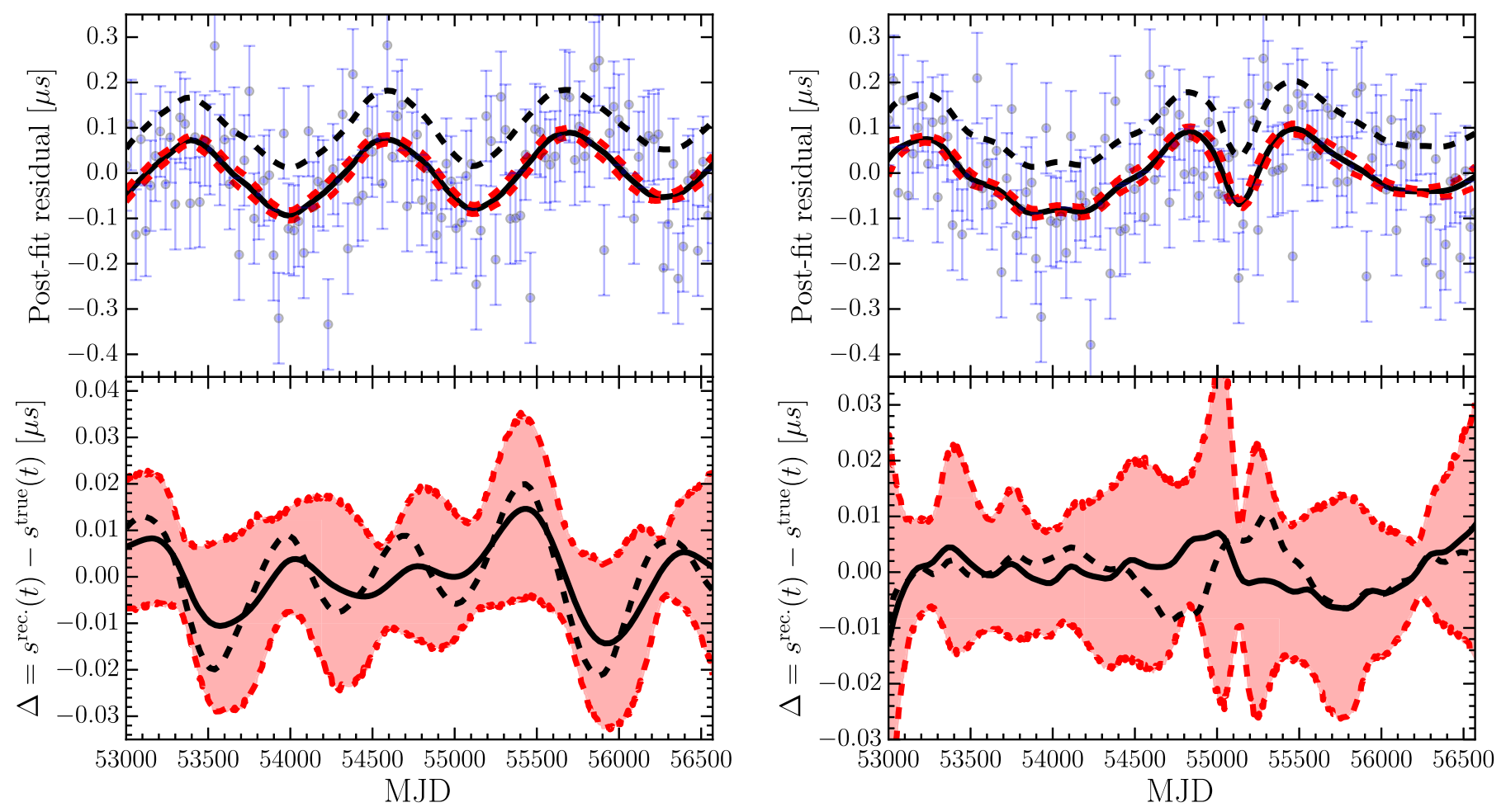

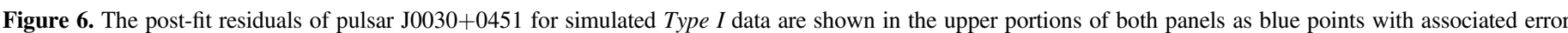

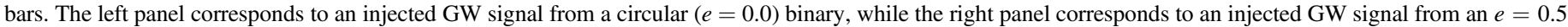

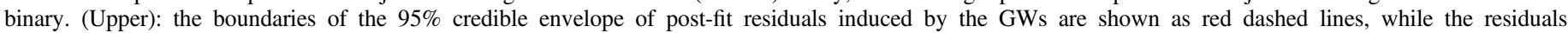

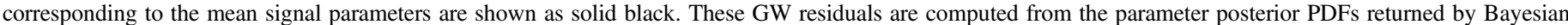

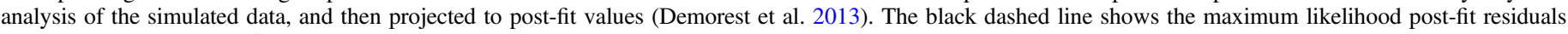

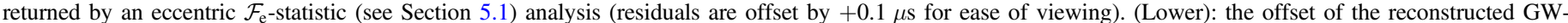

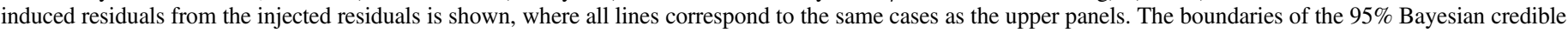
envelope of post-fit residuals encompasses $\Delta=0$, which is a good indicator of the robustness of the pipeline.

\subsection{Detection Prospects and Parameter Precision}

One might expect that distinctive high-frequency features due to periapsis passage (such as seen in Figure 6) may improve the prospects for detection. We investigate this by computing the optimal $\mathrm{S} / \mathrm{N}$ for a binary with varying orbital frequency, and a PTA timing baseline of 10 years in Type $I$ data. We draw the angular waveform parameters randomly and average over the resulting $\mathrm{S} / \mathrm{Ns}$. The result of this procedure as a function of binary eccentricity is shown in Figure 7, where we see a transition in behavior as the binary orbital frequency moves through the most sensitive location in the pulsar-timing band. From theoretical calculations and analysis of real data (Yardley et al. 2010; Arzoumanian et al. 2014; Moore et al. 2015), we expect the region of peak PTA sensitivity to a continuous GW to be at a GW frequency of $\sim 1 / T-2 / T$. Sensitivity is inhibited at lower frequencies by fitting of the pulsar quadratic spindown parameters in its timing-model, and higher frequencies are dominated by white TOA measurement errors. For $e=0$ binary signals in this simulated PTA, this peak corresponds to an orbital frequency of $\sim 1.6-3.2 \mathrm{nHz}$. In Figure 7 we see that at higher eccentricities the $\mathrm{S} / \mathrm{N}$ is enhanced when the injected orbital frequency lies below $1 \mathrm{nHz}$, and diminished when it lies above $5 \mathrm{nHz}$. We can make sense of this by recalling the spectral decomposition of the variance

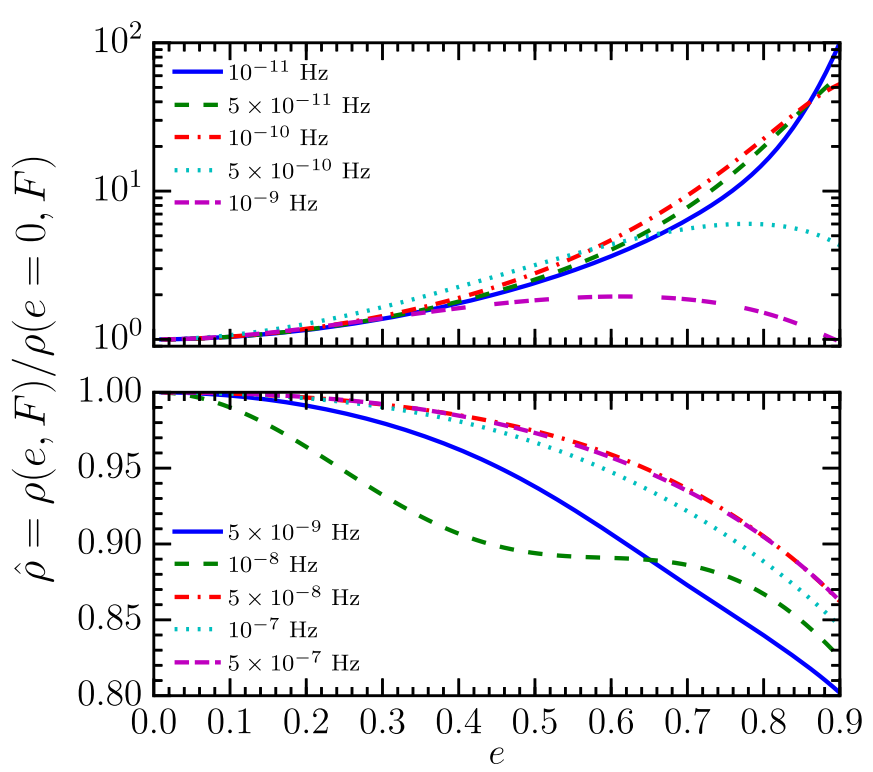

Figure 7. Normalized optimal $\mathrm{S} / \mathrm{N}$ of a single source as a function of the binary eccentricity for a PTA timing baseline of 10 years (Type I data). Only the Earth-term component is considered. Each curve corresponds to a different choice of binary orbital frequency, and is computed by averaging the $\mathrm{S} / \mathrm{N}$ over all waveform angular parameters. For reference, the GW frequency of greatest sensitivity in this Type I pulsar array is $\sim 5 \mathrm{nHz}$. 


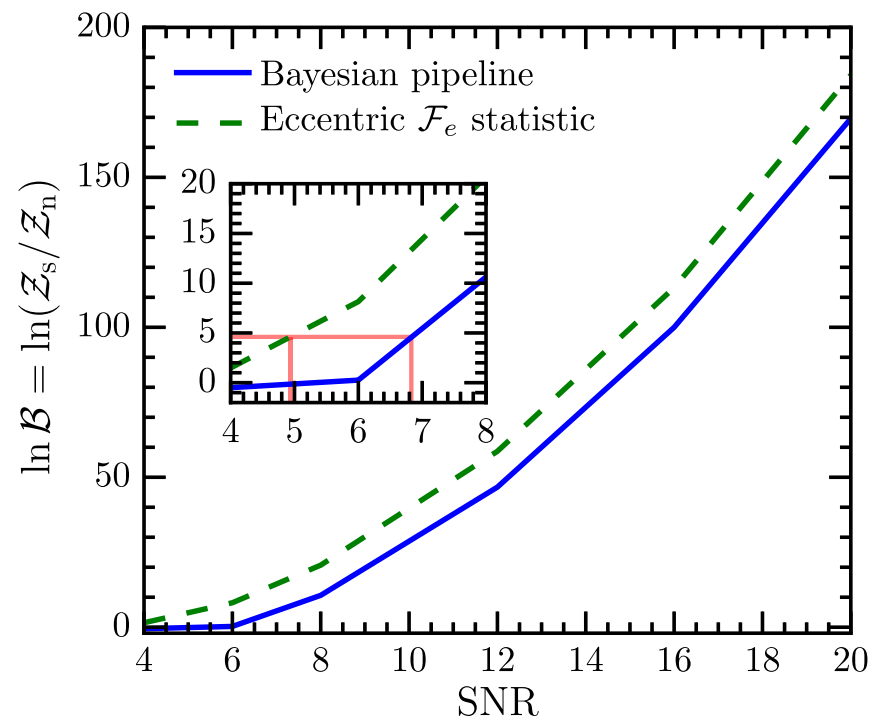

Figure 8. Bayes factors for a signal+noise model vs. a noise model alone in Type II data sets with varying $\mathrm{S} / \mathrm{N}$ injections. The injected binary parameters are the fiducial values given at the start of Section 5. The solid blue line shows the results for the full eccentric Bayesian pipeline, while the dashed green line shows the results for searches over the semi-maximized signal parameter space (intrinsic parameters) in the eccentric $\mathcal{F}_{\mathrm{e}}$ statistic. Red lines in the inset figure show the $\mathrm{S} / \mathrm{N}$ at which each technique reaches a Bayes factor of 100 .

of the GW-induced residuals shown in Figure 4, where as the eccentricity is increased the variance is distributed among higher harmonics of the orbital frequency. For systems with $F \lesssim 1 \mathrm{nHz}$ this will enhance the $\mathrm{S} / \mathrm{N}$ since power in the residual variance is shifted into the region of peak PTA sensitivity, while for systems with $F \gtrsim 5 \mathrm{nHz}$ this diminishes the $\mathrm{S} / \mathrm{N}$ since the power in the residual variance is distributed into higher, less sensitive frequencies of the PTA band.

With the approximate scaling behavior of $\mathrm{S} / \mathrm{N}$ with signal eccentricity established, we now investigate how $\mathrm{S} / \mathrm{N}$ maps to the Bayes factor of a signal+noise model versus a noise model alone. Bayesian model selection is actually carried out by computing the posterior odds ratio, which is the ratio of competing model evidences (Bayes factor, $\mathcal{B}$ ) multiplied by the prior odds ratio of each model. However, in the following we treat the latter quantity as being unity since in real searches we can not judge the a priori odds of a signal being in our data. Assuming fixed noise properties, the computation of the Bayes factor follows by integrating the likelihood ratio in Equation (27) over the signal parameter space. We judge a model to be favored over another if the Bayes factor exceeds 100 $(\ln \mathcal{B} \gtrsim 4.6)$.

We inject varying $\mathrm{S} / \mathrm{N}$ signals into noiseless Type II data sets with fiducial parameters and $e=0.5$. This eccentricity is a compromise between being a moderate value in our range of exploration, and (as seen later) where the discrimination between eccentric versus circular signal models is greatest. The injections are noiseless so that we can avoid the need to perform a large program of injections to average over noise realizations (Cornish 2010; Nissanke et al. 2010). There are some reservations over this approach in the low $\mathrm{S} / \mathrm{N}$ regime (Vallisneri 2011), however it is nevertheless correct at high $\mathrm{S} / \mathrm{N}$, so that we can consider the conclusions drawn here as optimistic but indicative of general trends. Importantly, in the following we verified that the peak of the recovered posterior distributions matched the injected signal parameters, which should be the case when the data sets are noiseless. This confirms that our techniques do not suffer as a result of the irregular sampling and heteroscedastic uncertainties associated with real data.

Figure 8 shows the growth of Bayes factors favoring a signal + noise model over a noise model alone in both the full signal parameter space (Bayesian pipeline) and intrinsic parameter space (eccentric $\mathcal{F}_{\mathrm{e}}$ statistic). Since the $\mathcal{F}_{\mathrm{e}}$ statistic is already maximized over half of the full signal parameter space, it has a lower search dimensionality than the full Bayesian pipeline and thus receives less of an Occam penalty. As seen in the inset of Figure 8 the $\mathcal{F}_{\mathrm{e}}$ statistic reaches a Bayesian detection threshold at $\mathrm{S} / \mathrm{N} \sim 5$ while the full Bayesian pipeline does so at $\mathrm{S} / \mathrm{N} \sim 7$.

An important question associated with $\mathrm{GW}$ detection is whether a threshold signal will be associated with any meaningful parameter measurement precisions. We address this issue by analyzing the widths of the $\{68 \%, 95 \%, 99.7 \%\}$ Bayesian credible regions with respect to the prior widths for binary orbital frequency, eccentricity, and sky location, at varying $\mathrm{S} / \mathrm{N}$. Our data sets are again of Type II with fiducial parameters and $e=0.5$. The results are shown in Figure 9, with measurement precisions obtained using the Bayesian pipeline along the top row, and precisions obtained with the eccentric $\mathcal{F}_{\mathrm{e}}$ statistic along the bottom row. At high $\mathrm{S} / \mathrm{N}$ the precisions for $\log _{10} F$ and $e$ obey a $1 / \mathrm{S} / \mathrm{N}$ scaling, and the sky location (being a compound of two parameters) obeys a $1 / \mathrm{S} / \mathrm{N}^{2}$ scaling. Both techniques perform comparably for $\mathrm{S} / \mathrm{N}>8$, however if we look at the width of the $99.7 \%$ credible region, we see that the eccentric $\mathcal{F}_{\mathrm{e}}$ statistic begins to update our prior knowledge of the parameter space at $\mathrm{S} / \mathrm{N} \gtrsim 5$, while this happens at $\mathrm{S} / \mathrm{N} \gtrsim 7$ for the full Bayesian pipeline. Hence, binary parameter measurement precisions become non-trivial once the Bayes factor favoring the presence of a signal exceeds our threshold value of 100. Rosado et al. (2015) investigated the likely properties of the first detectable continuous GW source in IPTA and SKA (Janssen et al. 2015) data, observing that the detection probability favored massive, nearby binaries with orbital frequencies $\lesssim 10 \mathrm{nHz}$. However, the authors did not consider eccentricity. From our results, we see that a threshold detection will provide an eccentricity measurement precision of $\sim 0.3$ (considering the $68 \%$ credible region width), which may allow the eccentricity to be sufficiently constrained as to perform inference on plausible environmental coupling influences (such as 3-body stellar scattering or circumbinary-disk interaction) which drove the binary's orbital evolution. Doing so will shed light on the astrophysics and environment of the binary's host galactic nucleus.

\subsection{Circular-model Penalty}

We now address the detection penalty one might incur by searching for an eccentric binary signal with a circular waveform model, which was also investigated in Zhu et al. (2015) using frequentist methods. We first investigate this using signal injections in Type I data for a variety of injected binary frequencies and eccentricities. The matched-filtering $\mathrm{S} / \mathrm{N}$ for a circular (monochromatic) template in data with an eccentric signal is computed, and compared to the optimal $\mathrm{S} / \mathrm{N}$ of the same eccentric signal. The resulting statistic, $\rho_{\text {circ }} / \rho_{\text {opt }}$, is a measure of the effectualness of the circular template in representing the eccentric signal (Buonanno et al. 2009). 

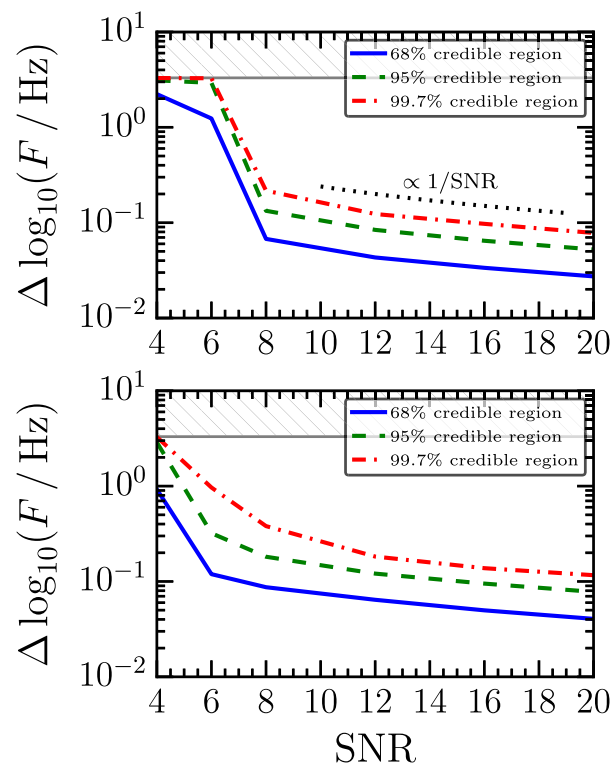
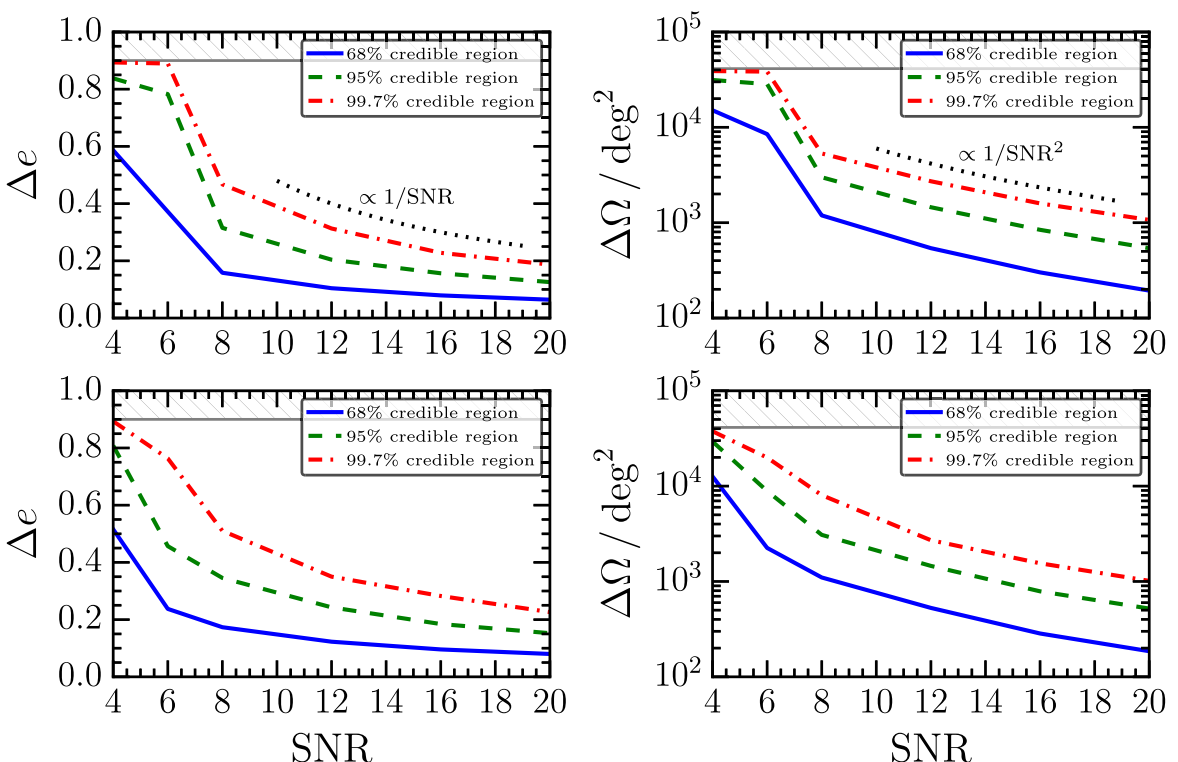

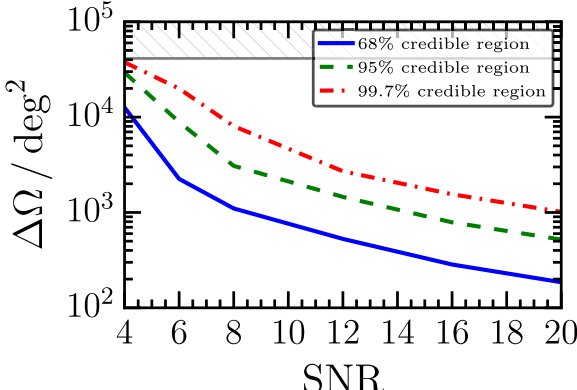

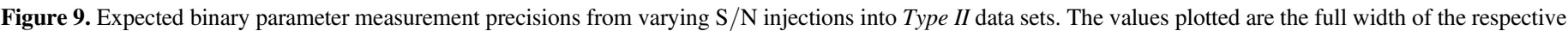

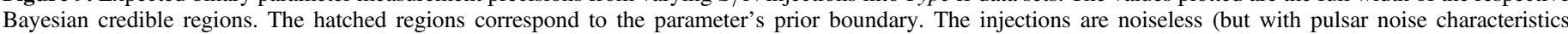

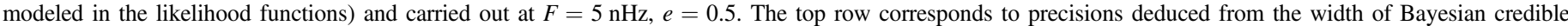

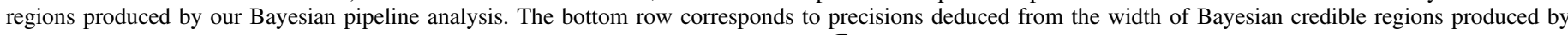
mapping the posterior distribution of the intrinsic parameter space with the eccentric $\mathcal{F}_{\mathrm{e}}$ statistic.

At each eccentricity, the $\mathrm{S} / \mathrm{N}$ is averaged over $10^{3}$ binary orientations and locations, and maximized over the frequency of a monochromatic template. The matched-filtering $\mathrm{S} / \mathrm{N}$ is computed in three different ways: (a) as a coherent $\mathrm{S} / \mathrm{N}$ for the entire pulsar array, maximized over the monochromatic template frequency; (b) as a coincident $\mathrm{S} / \mathrm{N}$, with the $\mathrm{S} / \mathrm{N}$ in each pulsar independently maximized over the monochromatic template frequency, and then added in quadrature to give the full array statistic; (c) as a coincident $\mathrm{S} / \mathrm{N}$, with the $\mathrm{S} / \mathrm{Ns}$ added in quadrature to give the full array statistic, but demanding a common frequency for the monochromatic template.

Our results for Type I data are shown in Figure 10 for orbital frequencies beyond the region of peak PTA sensitivity $(\gtrsim 5 \mathrm{nHz})$. For cases (a) and (b), the favored monochromatic template frequency is twice the orbital frequency until $e \sim 0.5-0.6$, and incurs an increasingly harsh $\mathrm{S} / \mathrm{N}$ penalty as the eccentricity of the signal is increased. However, beyond $e \sim 0.5-0.6$ the $\mathrm{S} / \mathrm{N}$ recovers slightly, since the template frequency now favors the fundamental harmonic of the signal, which is lower and closer to the region of peak PTA sensitivity. This is seen even more clearly in case (c), where there is a common monochromatic template frequency across all pulsars when constructing the coincident $\mathrm{S} / \mathrm{N}$. The loss in $\mathrm{S} / \mathrm{N}$ is slightly greater in (a) than in (b) and (c), since in the former we require signal coherence among all pulsars in the array. The behavior found for these three cases is likely pessimistic, since in real matched-filtering searches the $\mathrm{S} / \mathrm{N}$ is maximized over all template parameters rather than just the frequency. Lower orbital frequency $\mathrm{S} / \mathrm{N}$ curves exhibit similarly increasing penalties as the eccentricity is raised, but the trends are not as smooth.

We now investigate the circular-model penalty in terms of Bayesian model selection, by injecting fiducial signals of varying eccentricity and $\mathrm{S} / \mathrm{N}$ into Type II data. The results are shown in Figure 11, with the quoted Bayes factors corresponding to circular versus eccentric signal models. The top panel

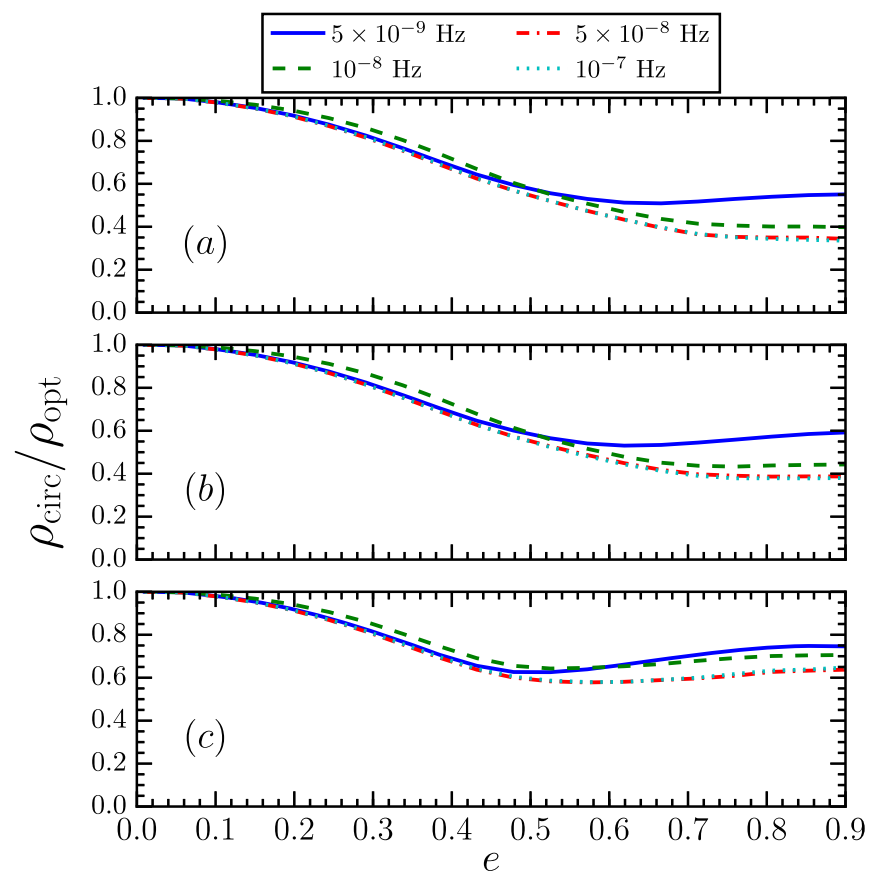

Figure 10. Ratio of circular-template matched-filtering $\mathrm{S} / \mathrm{N}$ to optimal $\mathrm{S} / \mathrm{N}$ for signals with various orbital frequencies and eccentricities. Case (a) shows results for a coherent array $\mathrm{S} / \mathrm{N}$. Case (b) shows results for a coincident array $\mathrm{S} / \mathrm{N}$ with independently maximized template frequencies in each pulsar. Case (c) shows results for a coincident array $\mathrm{S} / \mathrm{N}$ with a common monochromatic template frequency. Further details and discussion are provided in the text.

shows the results for the full Bayesian pipeline, while the bottom panel shows results for the eccentric $\mathcal{F}_{\mathrm{e}}$ statistic. Both techniques exhibit the same general trends: (1) at eccentricities $\lesssim 0.1$ the eccentric model receives an Occam penalty, resulting in the circular model being slightly favored, although not decisively so; (2) as the signal eccentricity increases so does the circular-model penalty, until the models are most easily 

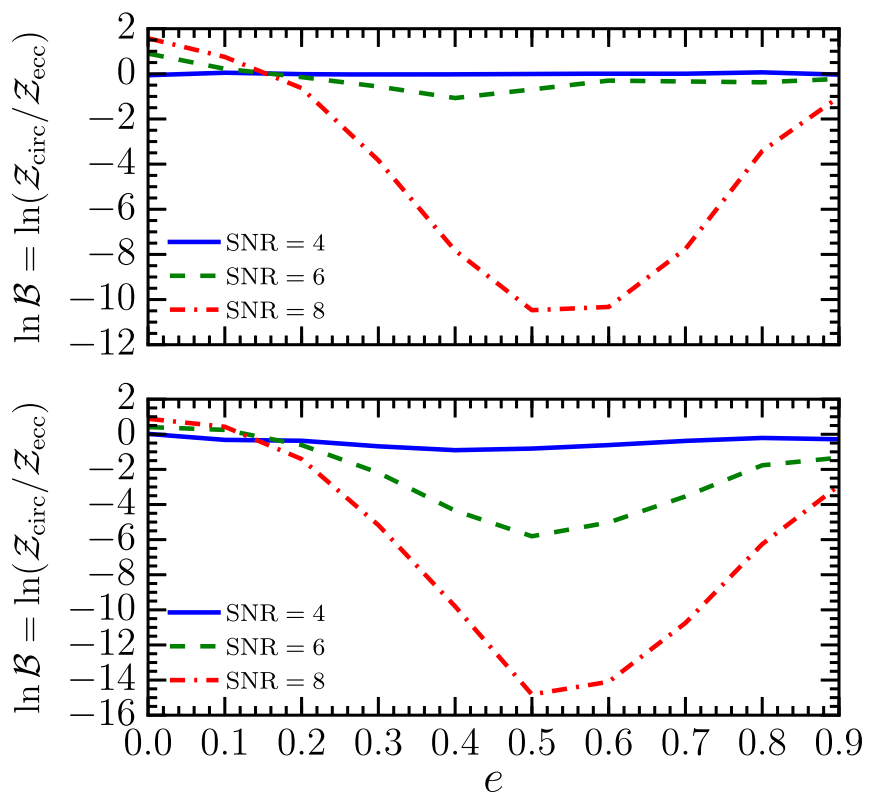

Figure 11. Bayes factors, $\mathcal{B}$, for circular vs. eccentric signal models when analyzing Type II data sets which have signals with varying injected eccentricity. The upper panel shows results for the full Bayesian analysis, while the lower panel shows results from mapping the posterior distribution of the intrinsic parameter space with the eccentric $\mathcal{F}_{\mathrm{e}}$ statistic.

discriminated at $e \sim 0.5-0.6$; (3) at higher eccentricities the signal model is being dominated by the fundamental harmonic, allowing the circular model to function as a better approximation to the injected signal than at intermediate eccentricities, resulting in a reduction in the circular-model penalty. These trends are qualitatively similar to those found by Zhu et al. (2015) in Figure 13 of their paper, however our eccentric search strategies exhibit superior performance at high eccentricity by virtue of modeling the distribution of signal harmonics with in-code adaptation (see Figure 2) rather than just including the lowest two harmonics. A key result of our analysis is that we require $\mathrm{S} / \mathrm{N}>8$ in order for an eccentric signal model to be correctly discriminated and favored when the true eccentricity is greater than $\sim 0.3$.

\section{CAVEATS AND FUTURE DIRECTIONS}

The analysis and results presented in this paper have relied on several assumptions. We discuss these here, and the prospects for relaxing these caveats in future work.

\subsection{Prospects for Including the Pulsar Term}

In the majority of this paper, we have ignored a full treatment of the pulsar term signal. Since the pulsar term is retarded with respect to the Earth term, it will represent the binary at an earlier stage of its orbital evolution, with a larger eccentricity and smaller orbital frequency. It is now well known that the pulsar term aids detection prospects for continuous wave sources, and is crucial in breaking degeneracies between the binary mass and its luminosity distance by providing extra information from the binary's evolution over the lag time between the Earth and pulsar term signals (Corbin \& Cornish 2010; Lee et al. 2011; Ellis 2013).

Being able to model the orbital evolution of the binary, and constrain the properties of this evolution through continuous GW searches with PTAs, will provide a unique opportunity to probe the influence of other non-GW driving mechanisms. For example, the rate at which the binary orbital frequency, $F$, is driven by GWs, stellar scattering, and circumbinary disk interactions, scales as $\propto F^{1 / 3}, \propto F^{1 / 3}, \propto F^{4 / 3}$, respectively (Sesana 2013). If we can include parametrized models of the rate of binary evolution in constructing full Earth and pulsar term signal models in a Bayesian or frequentist search, then we will be able to make statements about the relative importance of the aforementioned mechanisms. This in itself may provide clues as to how binaries are driven to sub-parsec orbital separations after dynamical friction in post-merger galaxies becomes inefficient, thereby adding to our knowledge of how the final parsec problem (Milosavljević \& Merritt 2003) is ameliorated.

For now, we estimate the degree to which employing only the Earth term in searches is sub-optimal for detection. We compute the matched-filter $\mathrm{S} / \mathrm{N}$ for an Earth term template applied to a full signal (including the pulsar term), and compare this to the optimal $\mathrm{S} / \mathrm{N}$ for the full signal. In constructing the pulsar term component of the signal, we evolve the orbital parameters of the binary backwards in time according to Equation (6) and the procedure outline in Section 4, where we assume all pulsars lie at a distance of $1 \mathrm{kpc}$ from the Earth. We assume all orbital evolution is GW driven. No pericenterdirection evolution or orbital-plane precession is considered, and we do not evolve the binary during the pulsar observation timespan of 10 years. To ease the computational burden, we use a sub-array of six pulsars spread across the sky, averaging the $\mathrm{S} / \mathrm{N}$ over $10^{3}$ binary locations and orientations. The observational cadences and timing baselines of the sub-array are of Type I variety.

The results are shown in Table 1 for a variety of Earth term orbital frequencies and eccentricities. As the orbital frequency and chirp mass are increased, the ratio of the Earth term $\mathrm{S} / \mathrm{N}$ to the full $\mathrm{S} / \mathrm{N}$ tends to grow with eccentricity. This is because higher mass, frequency, and eccentricity binaries are driven rapidly via $\mathrm{GW}$ emission, which in the most extreme cases leads to signals with pulsar term frequencies which are so far below the PTA sensitivity band that an Earth term template becomes an excellent approximation to the full signal. Even at fixed orbital frequency and eccentricity, the effect of increasing binary chirp mass is to raise the efficacy of an Earth term only template. However, care must be taken in the intermediate case, when we have moderate eccentricities, frequencies, and masses, which generate pulsar term signals that remain in the PTA band, and whose spectrum of GW frequencies may exceed the fundamental harmonic of the Earth term signal. The worst matches between signal and template occur for low mass, low eccentricity systems with orbital frequencies close to the region of peak PTA sensitvity $(\sim 5 \mathrm{nHz})$ - the combination of high array sensitivity and negligible orbital evolution leads to pulsar term signals close to this region of peak sensitivity, and thus very poor matches (which are sometimes negative since we employ a coherent $\mathrm{S} / \mathrm{N}$ ). In general, the pulsar term increases the signal detection prospects, but confusion may arise between different harmonics in the Earth and pulsar terms, which would harm parameter estimation efforts. So long as the Earth and pulsar terms remain distinguishable, we will learn more about the system parameters from the pulsar term's inclusion. Future work should study the prospects for incorporating the pulsar term in eccentric binary search strategies, and investigate the rich science that can be mined 
Table 1

The Matched-filter $\mathrm{S} / \mathrm{N}$ for an Earth Term Template is Compared Against the Optimal Full Signal $\mathrm{S} / \mathrm{N}$ to Construct $\rho_{\text {Earth }} / \rho_{\text {full }}$

\begin{tabular}{|c|c|c|c|c|c|c|c|c|c|c|c|c|c|c|c|c|c|c|c|c|}
\hline \multirow[t]{4}{*}{ Orbital Frequency (nHz) } & \multicolumn{20}{|c|}{ Eccentricity } \\
\hline & \multicolumn{4}{|c|}{$e=0.0$} & \multicolumn{4}{|c|}{$e=0.25$} & \multicolumn{4}{|c|}{$e=0.50$} & \multicolumn{4}{|c|}{$e=0.75$} & \multicolumn{4}{|c|}{$e=0.90$} \\
\hline & \multicolumn{4}{|c|}{$\mathcal{M}\left(M_{\odot}\right)$} & \multicolumn{4}{|c|}{$\mathcal{M}\left(M_{\odot}\right)$} & \multicolumn{4}{|c|}{$\mathcal{M}\left(M_{\odot}\right)$} & \multicolumn{4}{|c|}{$\mathcal{M}\left(M_{\odot}\right)$} & \multicolumn{4}{|c|}{$\mathcal{M}\left(M_{\odot}\right)$} \\
\hline & $10^{7}$ & $10^{8}$ & $10^{9}$ & $10^{10}$ & $10^{7}$ & $10^{8}$ & $10^{9}$ & $10^{10}$ & $10^{7}$ & $10^{8}$ & $10^{9}$ & $10^{10}$ & $10^{7}$ & $10^{8}$ & $10^{9}$ & $10^{10}$ & $10^{7}$ & $10^{8}$ & $10^{9}$ & $10^{10}$ \\
\hline 0.1 & 0.76 & 0.76 & 0.76 & 0.76 & 0.73 & 0.73 & 0.73 & 0.73 & 0.51 & 0.51 & 0.51 & 0.51 & 0.12 & 0.12 & 0.12 & 0.12 & 0.13 & 0.13 & 0.13 & 0.22 \\
\hline 0.5 & 0.78 & 0.78 & 0.78 & 0.78 & 0.66 & 0.66 & 0.66 & 0.66 & 0.33 & 0.33 & 0.33 & 0.34 & 0.15 & 0.15 & 0.16 & 0.41 & 0.07 & 0.07 & 0.36 & 0.87 \\
\hline 1.0 & 0.63 & 0.63 & 0.63 & 0.64 & 0.47 & 0.47 & 0.47 & 0.49 & 0.25 & 0.25 & 0.25 & 0.36 & 0.10 & 0.10 & 0.16 & 0.76 & 0.05 & 0.12 & 0.69 & 0.96 \\
\hline 5.0 & -0.03 & -0.03 & 0.07 & 0.65 & -0.02 & -0.02 & 0.13 & 0.72 & 0.0 & 0.01 & 0.38 & 0.87 & 0.02 & 0.16 & 0.81 & 0.99 & 0.11 & 0.71 & 0.99 & 1.0 \\
\hline 10.0 & -0.01 & 0.02 & 0.59 & 0.62 & -0.03 & 0.01 & 0.6 & 0.73 & -0.04 & 0.10 & 0.64 & 0.93 & 0.03 & 0.50 & 0.87 & 1.0 & 0.34 & 0.81 & 0.99 & 1.0 \\
\hline 50.0 & 0.21 & 0.68 & 0.63 & 0.42 & 0.3 & 0.68 & 0.56 & 0.95 & 0.53 & 0.67 & 0.81 & 0.99 & 0.68 & 0.66 & 0.99 & 1.0 & 0.60 & 0.97 & 1.0 & 1.0 \\
\hline 100.0 & 0.66 & 0.65 & 0.55 & 0.34 & 0.67 & 0.64 & 0.65 & 0.99 & 0.68 & 0.52 & 0.93 & 1.0 & 0.64 & 0.81 & 0.99 & 1.0 & 0.73 & 0.99 & 1.0 & 1.0 \\
\hline
\end{tabular}

Note. At each Earth term orbital frequency and eccentricity, we evolve a binary backwards in time by $L(1+\hat{\Omega} \cdot \hat{u})$ to construct the pulsar term waveform, where $L=1$ kpc for all pulsars. 


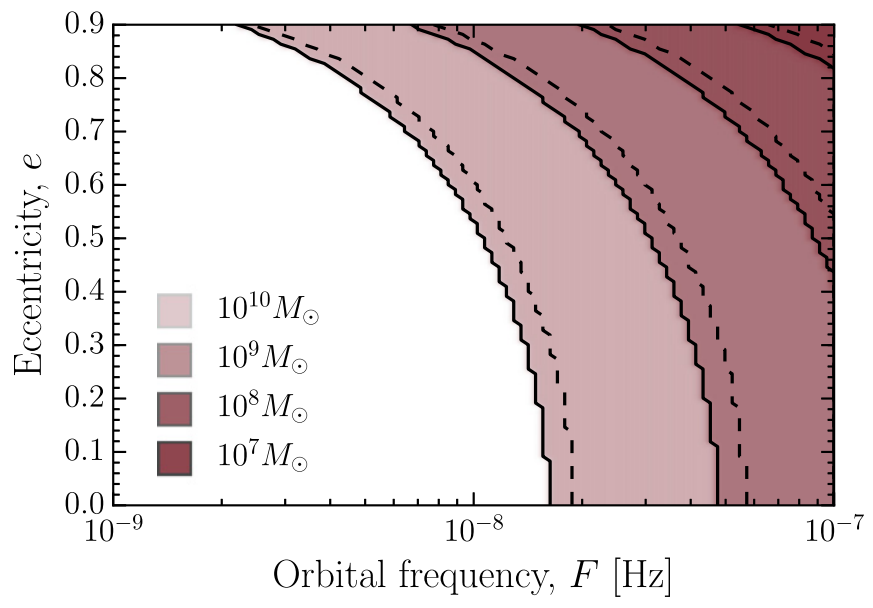

Figure 12. Exclusion regions in binary eccentricity and orbital frequency as a function of chirp mass, corresponding to parameter combinations where the fundamental (dashed black lines) and second harmonic (solid black lines on the boundary of shaded exclusion regions) of the orbital frequency evolve during $T=10$ years by more than the PTA frequency resolution, $\Delta f=1 / T=3.2 \mathrm{nHz}$, rendering the assumption of binary non-evolution invalid.

from having access to snapshots of the binary evolution from thousands of years in its past.

\subsection{Binary Orbital Evolution During the Observation Timespan}

We now test the assumption of binary non-evolution over typical PTA observation timespans. For different initial Earth term parameter choices $\{\mathcal{M}, F, e\}$, we numerically evolve a binary forward in time by 10 years according to Equation (6). Figure 12 shows exclusion regions in parameter space where the fundamental and second harmonic of the orbital frequency evolve by more than the PTA frequency resolution, $\Delta f=1 / T=3.2 \mathrm{nHz}$, which may render the approximation of binary non-evolution within our observing window invalid. The second harmonic will dominate the signal for low eccentricities while the fundamental harmonic will dominate at higher eccentricites.

A more rigorous way of testing this is to investigate how this assumption affects our ability to perform parameter estimation. If the non-evolution model performs well within the range of expected $\mathrm{S} / \mathrm{N}$, such that the systematic bias introduced via our assumption of binary non-evolution is smaller than statistical errors, then we can judge the model to be an excellent functioning approximation. More formally, we want to satisfy the indistinguishability criterion (Cutler \& Vallisneri 2007; Creighton \& Anderson 2012):

$$
(\delta s(t) \mid \delta s(t))<1,
$$

where $\delta s(t)$ corresponds to the difference between the approximated residuals in the non-evolution model and the true residuals. Satisfying the inequality in Equation (40) approximately corresponds to the systematic errors arising from modeling bias being smaller than statistical measurement errors. The tolerance $\mathrm{S} / \mathrm{N}, \rho_{\text {tol. }}$, above which systematic errors from insufficient template accuracy may exceed statistical measurement errors, and thus become problematic, is given by

$$
\rho_{\text {tol. }}^{2}=\frac{(s(t) \mid \boldsymbol{s}(t))}{(\delta \boldsymbol{s}(t) \mid \delta \boldsymbol{s}(t))}
$$

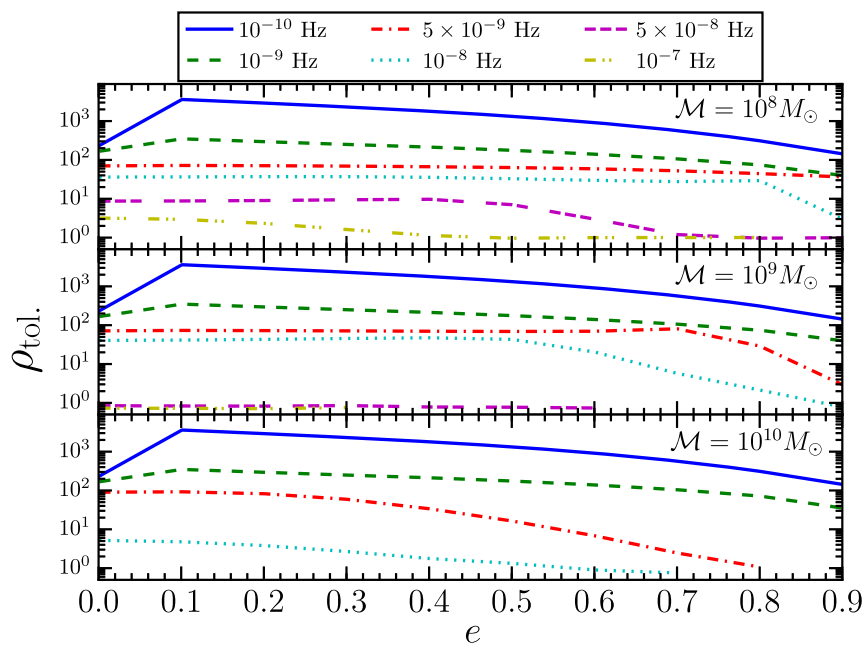

Figure 13. The tolerance $S / N, \rho_{\text {tol }}$, for a range of binary eccentricities, orbital frequencies, and chirp masses is shown. This indicates the $\mathrm{S} / \mathrm{N}$ above which systematic parameter errors (which occur by keeping binary parameters fixed over the 10 year PTA timing baseline) may exceed statistical measurement errors.

where $s(t)$ are the true residuals (concatenated over all pulsars) induced by a binary which may be evolving over our observation timespan. To compute this, we numerically evolve the orbital parameters of a binary over the 10 year timing baseline of a Type I data set using Equation (6), with varying choices of initial orbital frequency and eccentricity. The evolved orbit is then used to compute the pulse redshift and (via numerical integration) the $\mathrm{GW}$-induced timing residual at each pulse TOA. The typical ratio of the time required to compute the GW signal numerically versus analytically is $\sim \mathcal{O}\left(10^{4}\right)$, which is why a fully numerical approach is clearly intractable at present.

The tolerance $\mathrm{S} / \mathrm{N}$ is shown in Figure 13 as a function of binary eccentricity, orbital frequency, and chirp mass. We choose a cutoff value of the tolerance $\mathrm{S} / \mathrm{N}$ equal to 10 since this may correspond to realistic values of the $\mathrm{S} / \mathrm{Ns}$ of first PTA detections of single GW sources after $\sim 10$ years of IPTA and SKA1 activity (Rosado et al. 2015). If our model can be successfully applied to real signals above this cutoff value, then we conclude that the treatment used in this paper is valid well into the era of first PTA detections. We see that at a binary chirp mass of $10^{8} M_{\odot}$ the tolerance $\mathrm{S} / \mathrm{N}$ is above 10 for most frequencies and eccentricities, indicating that the assumption of non-evolution is valid. The approximation begins to break down at higher eccentricities and frequencies $\left(\gtrsim 5 \times 10^{-8} \mathrm{~Hz}\right)$ where the rate of binary evolution is higher. At $10^{9} M_{\odot}$ our model is appropriate at all eccentricities for frequencies lower than $10^{-8} \mathrm{~Hz}$, however the tolerance $\mathrm{S} / \mathrm{N}$ for $F=10^{-8} \mathrm{~Hz}$ drops below cutoff at $e \sim 0.7$, and at higher frequencies the assumption of binary non-evolution is inappropriate. Finally, for the most massive binaries with $\mathcal{M}=10^{10} M_{\odot}$, the tolerance $\mathrm{S} / \mathrm{N}$ remains above cutoff for orbital frequencies lower than $5 \times 10^{-9} \mathrm{~Hz}$ at all eccentricities, while at $5 \times 10^{-9} \mathrm{~Hz}$ the tolerance $\mathrm{S} / \mathrm{N}$ only drops below 10 at $e \sim 0.6$.

Therefore, our assumption (which has been shared by all other authors in this field) of binary non-evolution over typical PTA timing baselines is appropriate for most frequencies at or below the region of peak PTA sensitivity. The approximation 
only begins to break down for the most massive systems above orbital frequencies of $\sim 5 \times 10^{-9} \mathrm{~Hz}$ and eccentricities of 0.6 , allowing the signal model and analysis techniques developed in this article to be applied to real data with robust outcomes. Future studies are required to investigate faster and more tractable strategies for modeling the orbital evolution of high mass, high frequency, and high eccentricity binaries over PTA timing baselines.

\section{CONCLUSIONS}

PTAs are uniquely suited to explore the dynamical evolution of SMBHBs before and after they decouple from their astrophysical environments to become dominated by $\mathrm{GW}$ emission. An increasing number of studies tend to suggest that the mechanisms that may drive SMBHBs to small orbital separations could also lead to an increase in binary eccentricity that will be detectable in the frequency band of PTAs. Extracting this information from real data will substantially increase our understanding of the mechanisms that lead to the formation, hardening and eventual coalescence of SMBHBs. In this article we have introduced several tools to address this issue. We have developed a robust, accurate and computationally efficient Bayesian pipeline to explore the feasibility of detecting and reconstructing the astrophysical parameters of eccentric SMBHBs in PTA data, and have developed for the first time an eccentric $\mathcal{F}_{\mathrm{e}}$-statistic that is, by construction, suitable to study systems of arbitrary eccentricity.

We have used these tools to determine the accuracy with which a simulated eccentric signal could be reconstructed, and have conclusively shown that the recovered and injected parameters are completely consistent. Our prior knowledge of the eccentric binary parameter space will begin to be updated by data once the $\mathrm{S} / \mathrm{N}$ of the associated binary's GW signal exceeds $\sim 7$. We have also shown that the automated waveform generation algorithm, which determines the number of harmonics needed to ensure that the modeled GW signal reproduces the full numerical solution with an accuracy better than $99.999 \%$, prevents computational inefficiencies in the pipeline.

The influence of binary eccentricity on PTA single-source detection prospects was also considered. Assuming that the sensitivity peak of a PTA to continuous wave sources is located at a GW frequency $f_{0}$, we have shown that eccentricity will enhance the detection prospects of SMBHBs with orbital frequencies $\lesssim f_{0}$. This is because the signal spectrum of eccentric binaries is distributed into higher harmonics of the orbital frequency than in the case of a circular binary, leading to components of the signal being located in the region of maximum PTA sensitivity. On the other hand, binaries with orbital frequencies $\gtrsim f_{0}$ will undergo an $\mathrm{S} / \mathrm{N}$ attenuation because the signal power is shifted to higher frequencies where the PTA sensitivity is poorer and dominated by TOA measurement errors. In summary, systems with signals which are below band in the circular case get pushed into band through increasing eccentricity, while systems that are optimally located in frequency for the circular case get pushed out of band by eccentricity.

We found that applying a circular waveform model in the analysis of data with increasingly eccentric binary signals incurs an $\mathrm{S} / \mathrm{N}$ penalty which grows with eccentricity, and is $~$ $60 \%$ at worst case for coherent and coincident analyses. This was also investigated in a Bayesian context, where we found that $\mathrm{S} / \mathrm{Ns}$ greater than 8 are needed in order for an eccentric signal model to be correctly discriminated and favored over a circular signal model when the true signal eccentricity is $\geqslant 0.3$.

Several of the approximations used in the techniques presented in this article were briefly investigated. We found that for very high mass, frequency and eccentricity binaries, an Earth term signal model performs just as well as a full signal model incorporating the pulsar term, since the binary will have evolved so significantly that the pulsar term signal lies below band. Furthermore, the possible bias from assuming binary non-evolution over a PTA observation time of 10 years was studied, and was found to be unimportant for moderately massive and eccentric systems in the era of first PTA detections.

There are several topics in continuous GW searches that should be addressed in the near future, including the need to assess the possible covariances involved in simultaneous continuous GW searches and stochastic GW background searches. One can imagine that the reduction in low-frequency sensitivity associated with having fit for the pulsar quadratic spindown parameters will be exacerbated by the concurrent search for a stochastic GW background signal dominated by low-frequency power. Also, the possibility of having multiple resolvable continuous-wave sources may lead to difficulties in isolating each source during the Bayesian searches, resulting in interesting covariances. However there are ongoing efforts to resolve this issue (J. Ellis 2015, private communication). Furthermore, as new data is added to each PTA and combined to form IPTA data sets, the prospects for continuous GW source detection grow stronger. Current pipelines should be tested against signals injected into near-future type data sets as a means to inform new advances in analysis procedures. Finally, the efficacy of performing continuous GW searches on data sets having signals composed of realistic GW source populations must be addressed in the near future. These areas of future study have not yet been investigated with circularbinary GW signal models, however our development in this paper of more complete signals models which include eccentricity will endow these studies with greater verisimilitude.

Huerta et al. (2015) and this article have provided a solid foundation to explore in a consistent way the influence of eccentricity on the detection and parameter estimation of SMBHBs with PTAs. The tools presented in this article can be readily incorporated into all present and planned analysis pipelines. The toolkit introduced in these articles could be extended to explore in detail what constraints may be placed on the various astrophysical mechanisms that can drive the dynamical evolution of SMBHBs prior to becoming dominated by $\mathrm{GW}$ emission.

We thank the anonymous referee for their remarks, which significantly improved the depth and quality of this manuscript. This research was in part supported by SRT's appointment to the NASA Postdoctoral Program at the Jet Propulsion Laboratory, administered by Oak Ridge Associated Universities through a contract with NASA. J. R. G.'s work is supported by the Royal Society. We thank Justin Ellis for useful feedback on this manuscript, and Alberto Sesana for fruitful discussions. We also thank the anonymous referee for many helpful suggestions which significantly improved this manuscript. This work was supported in part by National 
Science Foundation Grant No. PHYS-1066293 and the hospitality of the Aspen Center for Physics. We are grateful for computational resources provided by the Leonard E Parker Center for Gravitation, Cosmology and Astrophysics at University of Wisconsin-Milwaukee. NANOGrav is supported by a Physics Frontier Center award (PHY-1430284) from the National Science Foundation.

\section{REFERENCES}

Anholm, M., Ballmer, S., Creighton, J. D. E., Price, L. R., \& Siemens, X. 2009, PhRvD, 79, 084030

Apostolatos, T. A., Cutler, C., Sussman, G. J., \& Thorne, K. S. 1994, PhRvD, 49,6274

Arzoumanian, Z., Brazier, A., Burke-Spolaor, S., et al. 2014, ApJ, 794, 141

Arzoumanian, Z., Brazier, A., Burke-Spolaor, S., et al. 2015, arXiv:1508.03024

Babak, S., \& Sesana, A. 2012, PhRvD, 85, 044034

Barack, L., \& Cutler, C. 2004, PhRvD, 69, 082005

Berger, E. 2013, arXiv:1306.3960

Berger, E., Fong, W., \& Chornock, R. 2013, ApJL, 774, L23

Book, L. G., \& Flanagan, É É 2011, PhRvD, 83, 024024

Buchner, J., Georgakakis, A., Nandra, K., et al. 2014, A\&A, 564, A125

Buonanno, A., Iyer, B. R., Ochsner, E., Pan, Y., \& Sathyaprakash, B. S. 2009, PhRvD, 80, 084043

Chandrasekhar, S. 1983, The Mathematical Theory of Black Holes, Vol. 69 (Oxford/New York: Clarendon Press/Oxford Univ. Press)

Corbin, V., \& Cornish, N. J. 2010, arXiv:1008.1782

Cornish, N. J. 2010, arXiv:1007.4820

Cornish, N. J., \& Porter, E. K. 2007, CQGra, 24, 5729

Creighton, J. D., \& Anderson, W. G. 2012, Gravitational-Wave Physics and Astronomy: An Introduction to Theory, Experiment and Data Analysis (New York: Wiley)

Cutler, C., \& Vallisneri, M. 2007, PhRvD, 76, 104018

Demorest, P. B., Ferdman, R. D., Gonzalez, M. E., et al. 2013, ApJ, 762, 94

Edwards, R. T., Hobbs, G. B., \& Manchester, R. N. 2006, MNRAS, 372, 1549

Ellis, J. A. 2013, CQGra, 30, 224004

Ellis, J. A., Siemens, X., \& Creighton, J. D. E. 2012, ApJ, 756, 175

Feroz, F., \& Hobson, M. P. 2008, MNRAS, 384, 449

Feroz, F., Hobson, M. P., \& Bridges, M. 2009, MNRAS, 398, 1601

Feroz, F., Hobson, M. P., Cameron, E., \& Pettitt, A. N. 2013, arXiv:1306.2144

Finn, L. S. 2001, PhRvD, 63, 102001

Foster, R. S., \& Backer, D. C. 1990, ApJ, 361, 300

Gebhardt, K., Kormendy, J., Ho, L., et al. 2000, ApJL, 543, L5

Goldstein, H. 1950, Classical Mechanics (Reading, MA: Addison-Wesley)

Hobbs, G. 2013, CQGra, 30, 224007

Hobbs, G. B., Edwards, R. T., \& Manchester, R. N. 2006, MNRAS, 369, 655

Huerta, E. A., McWilliams, S. T., Gair, J. R., \& Taylor, S. R. 2015, PhRvD, 92, 063010

Hughes, S. A. 2009, ARA\&A, 47, 107

Hughes, S. A., \& Blandford, R. D. 2003, ApJL, 585, L101

Jaffe, A. H., \& Backer, D. C. 2003, ApJ, 583, 616

Janka, H.-T., Eberl, T., Ruffert, M., \& Fryer, C. L. 1999, ApJL, 527, L39
Janssen, G. H., et al. 2015, in Proc. Advancing Astrophysics with the Square Kilometre Array (AASKA14), 37

Khan, F. M., Preto, M., Berczik, P., et al. 2012, ApJ, 749, 147

Kormendy, J., \& Richstone, D. 1995, ARA\&A, 33, 581

Kramer, M., \& Champion, D. J. 2013, CQGra, 30, 224009

Lee, K. J., Wex, N., Kramer, M., et al. 2011, MNRAS, 414, 325

Lee, W. H., \& Ramirez-Ruiz, E. 2007, NJPh, 9, 17

Magorrian, J., Tremaine, S., Richstone, D., et al. 1998, AJ, 115, 2285

Manchester, R. N. \& IPTA 2013, CQGra, 30, 224010

McLaughlin, M. A. 2013, CQGra, 30, 224008

Metzger, B. D., \& Berger, E. 2012, ApJ, 746, 48

Milosavljević, M., \& Merritt, D. 2003, ApJ, 596, 860

Mingarelli, C. M. F., Grover, K., Sidery, T., Smith, R. J. E., \& Vecchio, A. 2012, PhRvL, 109, 081104

Misner, C. W., Thorne, K. S., \& Wheeler, J. A. 1973, Gravitation (San Francisco, CA: Freeman)

Moore, C. J., Taylor, S. R., \& Gair, J. R. 2015, CQGra, 32, 055004

NANOGrav Collaboration, Arzoumanian, Z., Brazier, A., et al. 2015, ApJ, 813,65

Nissanke, S., Holz, D. E., Hughes, S. A., Dalal, N., \& Sievers, J. L. 2010, ApJ, 725, 496

Peters, P. C. 1964, PhRv, 136, B1224

Peters, P. C., \& Mathews, J. 1963, PhRvD, 131, 435

Peterson, B. M., Ferrarese, L., Gilbert, K. M., et al. 2004, ApJ, 613, 682

Pierro, V., Pinto, I. M., Spallicci, A. D., Laserra, E., \& Recano, F. 2001, MNRAS, 325, 358

Piran, T., Nakar, E., \& Rosswog, S. 2013, MNRAS, 430, 2121

Rajagopal, M., \& Romani, R. W. 1995, ApJ, 446, 543

Roedig, C., Dotti, M., Sesana, A., Cuadra, J., \& Colpi, M. 2011, MNRAS, 415, 3033

Roedig, C., \& Sesana, A. 2012, JPhCS, 363, 012035

Rosado, P. A., Sesana, A., \& Gair, J. 2015, MNRAS, 451, 2417

Sesana, A. 2010, ApJ, 719, 851

Sesana, A. 2013, CQGra, 30, 224014

Sesana, A., Gualandris, A., \& Dotti, M. 2011, MNRAS, 415, L35

Sesana, A., Haardt, F., \& Madau, P. 2008, ApJ, 686, 432

Sesana, A., \& Vecchio, A. 2010a, CQGra, 27, 084016

Sesana, A., \& Vecchio, A. 2010b, PhRvD, 81, 104008

Sesana, A., Vecchio, A., \& Volonteri, M. 2009, MNRAS, 394, 2255

Soltan, A. 1982, MNRAS, 200, 115

Tanvir, N. R., Levan, A. J., Fruchter, A. S., et al. 2013, Natur, 500, 547

Taylor, S., Ellis, J., \& Gair, J. 2014, PhRvD, 90, 104028

Thorne, K. S. 1987, in Three Hundred Years of Gravitation, ed. S. Hawking, \& W. Israel (Cambridge: Cambridge Univ. Press)

Vallisneri, M. 2011, PhRvL, 107, 191104

van Haasteren, R., Levin, Y., McDonald, P., et al. 2009, MNRAS, 395, 1005 van Haasteren, R., \& Vallisneri, M. 2014, PhRvD, 90, 104012

Vasiliev, E., Antonini, F., \& Merritt, D. 2015, ApJ, 810, 49

Watson, G. 1995, A Treatise on the Theory of Bessel Functions (Cambridge: Cambridge Univ. Press)

Wyithe, J. S. B., \& Loeb, A. 2003, ApJ, 590, 691

Yardley, D. R. B., Hobbs, G. B., Jenet, F. A., et al. 2010, MNRAS, 407, 669

Yunes, N., Arun, K. G., Berti, E., \& Will, C. M. 2009, PhRvD, 80, 084001

Zhu, X.-J., Wen, L., Hobbs, G., et al. 2015, MNRAS, 449, 1650 